\title{
Water-anion hydrogen bonding dynamics: Ultrafast IR experiments and simulations
}

\author{
Steven A. Yamada, ${ }^{1}$ Ward H. Thompson, ${ }^{2, a)}$ and Michael D. Fayer ${ }^{1, b)}$ \\ ${ }^{1}$ Department of Chemistry, Stanford University, Stanford, California 94305, USA \\ ${ }^{2}$ Department of Chemistry, University of Kansas, Lawrence, Kansas 66045, USA
}

(Received 16 March 2017; accepted 18 May 2017; published online 15 June 2017)

\begin{abstract}
Many of water's remarkable properties arise from its tendency to form an intricate and robust hydrogen bond network. Understanding the dynamics that govern this network is fundamental to elucidating the behavior of pure water and water in biological and physical systems. In ultrafast nonlinear infrared experiments, the accessible time scales are limited by water's rapid vibrational relaxation (1.8 ps for dilute $\mathrm{HOD}$ in $\mathrm{H}_{2} \mathrm{O}$ ), precluding interrogation of slow hydrogen bond evolution in non-bulk systems. Here, hydrogen bonding dynamics in bulk $\mathrm{D}_{2} \mathrm{O}$ were studied from the perspective of the much longer lived (36.2 ps) CN stretch mode of selenocyanate $\left(\mathrm{SeCN}^{-}\right)$using polarization selective pump-probe (PSPP) experiments, two-dimensional infrared (2D IR) vibrational echo spectroscopy, and molecular dynamics simulations. The simulations make use of the empirical frequency mapping approach, applied to $\mathrm{SeCN}^{-}$for the first time. The PSPP experiments and simulations show that the orientational correlation function decays via fast $(2.0 \mathrm{ps})$ restricted angular diffusion (wobbling-ina-cone) and complete orientational diffusive randomization ( $4.5 \mathrm{ps}$ ). Spectral diffusion, quantified in terms of the frequency-frequency correlation function, occurs on two time scales. The initial $0.6 \mathrm{ps}$ time scale is attributed to small length and angle fluctuations of the hydrogen bonds between water and $\mathrm{SeCN}^{-}$. The second 1.4 ps measured time scale, identical to that for $\mathrm{HOD}$ in bulk $\mathrm{D}_{2} \mathrm{O}$, reports on the collective reorganization of the water hydrogen bond network around the anion. The experiments and simulations provide details of the anion-water hydrogen bonding and demonstrate that $\mathrm{SeCN}^{-}$ is a reliable vibrational probe of the ultrafast spectroscopy of water. Published by AIP Publishing. [http://dx.doi.org/10.1063/1.4984766]
\end{abstract}

\section{INTRODUCTION}

Water hydrogen bonding governs the structure and dynamics of a vast range of chemical systems ranging from geology to biology. Ultrafast infrared (IR) spectroscopy is uniquely suited to study the dynamics of the water hydrogen bond network. The reorientation of water and the randomization of the liquid structure occur on sub-picosecond and picosecond time scales, which can be well-resolved by ultrafast IR experiments. In contrast to electronic excitations, vibrational transitions are minimally perturbative to the nuclear coordinates of the molecule, making it possible to observe the thermal equilibrium dynamics that underlie hydrogen bond dissociation and formation.

Infrared polarization selective pump-probe (PSPP) experiments have shown that the orientational relaxation of the $\mathrm{OH}$ transition dipole of dilute HOD in bulk $\mathrm{D}_{2} \mathrm{O}$ occurs on a 3 ps time scale, ${ }^{1-3}$ in general agreement with the rotational correlation times from $\mathrm{NMR}^{4}$ and dielectric relaxation ${ }^{5}$ studies on $\mathrm{D}_{2} \mathrm{O}$. The spectral diffusion of HOD (and other solutes) in water has also remained a subject of fundamental interest to spectroscopists and theoreticians as the accurate prediction of dynamic observables informs the development of meaningful liquid models. ${ }^{6-10}$ Spectral diffusion refers to

\footnotetext{
a)Email: wthompson@ku.edu. Telephone: 785 864-3980.

b) Email: fayer@stanford.edu. Telephone: 650 723-4446.
}

the process by which an ensemble of vibrational oscillators progressively loses memory of its initial frequency distribution. Since the vibrational frequency of a probe molecule is coupled to the motions of solvent molecules in the hydrogen bond network, measurement of the frequency-frequency correlation function (FFCF) of the ensemble reveals the fundamental time scales associated with these structural motions. ${ }^{11}$ Ultrafast two-dimensional infrared (2D IR) vibrational echo experiments on the OD stretch of dilute $\mathrm{HOD}$ in $\mathrm{H}_{2} \mathrm{O}$ gave a $0.4 \pm 0.1$ ps fast component followed by a $1.7 \pm 0.2$ ps component. ${ }^{6,12-14}$ The fast time scale arises from local hydrogen bond length and angle fluctuations, followed by the slower relaxation arising from concerted rearrangement of the extended hydrogen bond network. ${ }^{1,6,12,15}$ Vibrational echo peak shift (PS) experiments revealed that the spectral diffusion of the $\mathrm{OH}$ stretch of dilute $\mathrm{HOD}$ in $\mathrm{D}_{2} \mathrm{O}$ proceeds with very similar time scales. ${ }^{1,15}$ The slow component is $1.4 \pm 0.2 \mathrm{ps}$. The integrated correlation time, obtained from the integral of the FFCF, gave a value of $\sim 0.34 \mathrm{ps}$. The integral included a very fast oscillatory feature observed at the shortest times.

The study of hydrogen bond dynamics via ultrafast IR spectroscopy and molecular dynamics (MD) simulations has been extended from pure water to aqueous salt solutions, , 10,13,16-22 which are ubiquitous in chemistry and biology. These studies probed the dynamics of water in dilute and concentrated aqueous systems. However, many of the ionic 
solutes used in the studies were not vibrationally active and therefore did not present the opportunity to study dynamics in aqueous solutions from the perspective of the ions. Two exceptions were cyanide ${ }^{9}$ and borohydride, ${ }^{22}$ although both presented limitations. Cyanide is an incredibly weak absorber, which made the acquisition of high signal-to-noise data challenging. In the latter case, the triple degeneracy of the $\mathrm{B}-\mathrm{H}$ stretch and the chemical instability of borohydride in water complicated the results.

In the following, we present ultrafast IR experiments and MD simulations that characterize the reorientation and spectral diffusion of the selenocyanate anion $\left(\mathrm{SeCN}^{-}\right)$in dilute aqueous solution, 0.1-0.3 M KSeCN in $\mathrm{D}_{2} \mathrm{O}$. In this concentration range, each $\mathrm{SeCN}^{-}$is surrounded by multiple $\mathrm{D}_{2} \mathrm{O}$ solvation shells ( $\sim 185$ waters per anion), and its dynamics are determined by motions of the surrounding water hydrogen bond network. $\mathrm{SeCN}^{-}$is a useful vibrational probe for a number of reasons. First, the $\mathrm{CN}$ stretch is well approximated as a local mode, which simplifies the interpretation of solvent dynamics sensed by the vibration. Second, the transition dipole direction, which establishes directionality in the experiments and simulations, can be taken as the $\mathrm{CN}$ bond unit vector. Third, $\mathrm{SeCN}^{-}$ has a significantly longer vibrational lifetime than either the $\mathrm{OH}$ or OD stretches of HOD. As we will show here, $\mathrm{SeCN}^{-}$ dynamics, particularly spectral diffusion, reliably probe essential features of the water hydrogen bond network. This presents the opportunity to study hydrogen bond dynamics in systems in which the HOD lifetime is limiting, principally systems in which the water dynamics are expected to be slow compared to bulk water. Water confined in mesoporous silica with pore diameters of a few nanometers is one important example. ${ }^{23,24}$

Fast time scales in both the orientational relaxation and spectral diffusion of $\mathrm{SeCN}^{-}$were resolved in the experiments. In contrast to HOD, which displays 3 ps single exponential orientational relaxation dynamics, the $\mathrm{SeCN}^{-}$anion exhibits fast restricted orientational diffusion in a small cone of angles in addition to diffusive randomization on a $4.5 \mathrm{ps}$ time scale. The spectral diffusion, measured with 2D IR and quantified in terms of the FFCF, has an $\sim 600$ fs component. The simulations indicate that, like the $\sim 400 \mathrm{fs}$ component in the FFCF of HOD, the 600 fs component originates from local hydrogen bond dynamics among $\mathrm{CN}$ and water involving small length and angle fluctuations in relatively fixed hydrogen bonding configurations about the $\mathrm{SeCN}^{-}$anion. The long time scale component, $1.4 \mathrm{ps}$, is identical to the final component of the FFCF of HOD. The results reinforce the picture that $\mathrm{SeCN}^{-}$ is a reliable probe of the local hydrogen bond fluctuations and the global rearrangement dynamics that randomize the water hydrogen bond network.

\section{EXPERIMENTAL PROCEDURES}

\section{A. Sample preparation and linear IR spectroscopy}

Potassium selenocyanate ( $99 \%$ purity) and deuterium oxide (100.0 at. \% D) were purchased from Fischer Scientific (Acros Organics brand). KSeCN was dried on a Schlenk line under vacuum $(\sim 100 \mathrm{mTorr})$ at $60{ }^{\circ} \mathrm{C}$ for one week and stored in a nitrogen glovebox. Samples were prepared by dissolving the dry $\mathrm{KSeCN}$ in $\mathrm{D}_{2} \mathrm{O}$ at 0.1 and $0.3 \mathrm{M}$ concentrations. The solution concentrations were kept low to ensure that vibrational excitation transfer between anions was statistically improbable. ${ }^{25,26}$ Sample cells used in the experiments were assembled by sandwiching the solutions between two $3 \mathrm{~mm}$ thick, 1 in diameter $\mathrm{CaF}_{2}$ windows separated by a Teflon spacer. The spacer thickness was varied between 25 and $56 \mu \mathrm{m}$ to achieve a sample absorbance of $\sim 0.2-0.3$. Fourier Transform IR (FT IR) spectra were measured with a Thermo Scientific Nicolet 6700 FT IR spectrometer purged with air scrubbed free of $\mathrm{CO}_{2}$ and $\mathrm{H}_{2} \mathrm{O}$. The absorption spectra of pure $\mathrm{D}_{2} \mathrm{O}$ with identical spacer thicknesses were measured and subtracted from the sample spectra, yielding the spectra of the $\mathrm{CN}$ stretch.

\section{B. Laser system and optical setup}

The 2D IR pulse-shaping spectrometer has been described in detail in a previous publication. ${ }^{27}$ Briefly, a Ti:sapphire oscillator seeds a regenerative amplifier that outputs $650 \mu \mathrm{J}$ pulses at $1 \mathrm{kHz}$ centered at $800 \mathrm{~nm}$ with a $100 \mathrm{fs}$ full-width at half maximum (FWHM) duration. The regen output pumps an optical parametric amplifier/difference frequency generation (OPA/DFG) system, resulting in $150 \mathrm{fs}\left(\sim 110 \mathrm{~cm}^{-1} \mathrm{FWHM}\right.$ bandwidth) mid-IR pulses centered at $2075 \mathrm{~cm}^{-1}$ with an energy of $6 \mu \mathrm{J}$. The 2D IR spectrometer is configured in a pump-probe geometry. ${ }^{11,27,28}$ The mid-IR pulses are split into a strong pump and weaker probe pulse in 92:8 intensity ratio with a $\mathrm{ZnSe}$ beam splitter. The strong pump pulse is directed into a mid-IR frequency-domain pulse-shaper consisting of a germanium acousto-optic modulator (AOM) at the Fourier plane of a 4- $f$ pulse-shaping geometry. ${ }^{27-29}$ The number, amplitude, phase, and temporal delay of the pump pulse(s) that emerge at the output are precisely controlled by the AOM. The weaker probe pulse is sent through a precision mechanical delay stage that sets the time delay between the pump and the probe. The two beams are then focused and spatially overlapped in the sample. In the pump-probe geometry, the pump-probe and vibrational echo signals are emitted collinearly with the probe pulse. ${ }^{11,27,28}$ After the sample, the probe/signal is sent into a monochromator acting as a spectrograph and detected with a liquid nitrogen cooled 32-element $\mathrm{HgCdTe}$ (MCT) IR array detector.

\section{Polarization selective pump-probe experiments}

Detailed discussions on the theory and practical implementation of mid-IR PSPP experiments can be found elsewhere. ${ }^{30,31}$ The experimental pulse sequence involves a single pump pulse and a probe pulse separated by a delay, $t$. The pump is chopped at half the $1 \mathrm{kHz}$ laser repetition rate so that the probe spectrum can be subtracted from the PP signal, which is then divided by (normalized to) the probe spectrum. The polarizations of the pump and probe are established directly before the sample. The polarization of the probe is fixed to horizontal $\left(0^{\circ}\right.$ or parallel to the plane of the optical table) with a silicon wire-grid polarizer. A halfwave plate followed by a polarizer fixes the pump polarization to $45^{\circ}$ relative to the probe polarization. Directly after the sample, a polarizer in a computer controlled rotation mount resolves the probe at $+45^{\circ}$ or $-45^{\circ}$, corresponding to 
polarizations parallel and perpendicular to the pump, respectively. Finally, the parallel and perpendicular signals, $S_{\|}(t)$ and $S_{\perp}(t)$, are projected back to horizontal $\left(0^{\circ}\right)$ polarization by a second polarizer before the entrance slit of the spectrograph. The final polarizer ensures that both signals encounter the same grating efficiency, which is polarization dependent. The component frequencies are spectrally dispersed and detected.

\section{2D IR spectroscopy}

In the 2D IR experiment, two pump pulses (1 and 2) and one probe pulse (3) interact with the sample, resulting in the emission of the 3rd order vibrational echo signal in the phase matched direction, i.e., collinear with pulse $3 .^{11,14}$ The time between pulses 1 and 2, between 2 and 3, and following 3 is the first coherence period, $\tau$, the population period, $T_{w}$, and the final coherence period, $t$. The following qualitative description is useful in understanding the experiment. Pump pulses 1 and 2 label the ensemble of $\mathrm{SeCN}^{-}$vibrational oscillators with their initial frequencies during the first coherence period. In the $T_{w}$ period, structural evolution of the solvating water causes the vibrational frequencies to change. Arrival of pulse 3 stimulates the emission of the vibrational echo, which reads out the final frequency distribution of the ensemble that arises from configurational evolution in the liquid occurring over the $T_{w}$ period.

The phase matching condition for the pump-probe geometry results in the vibrational echo signal being emitted collinearly with pulse $3 .^{27,28}$ Pulse 3 serves as a local oscillator (LO) that interferes with the echo signal, providing information on its phase. The combined echo/LO is sent to the same spectrograph and array detector as in the PSPP experiments. The spectrograph performs a Fourier transform of the combined echo/LO pulse with respect to $t$, giving the $\omega_{\mathrm{m}}$ (vertical) axis of the 2D spectrum. To obtain the horizontal axis, at each $T_{w}$, the delay $\tau$ is scanned, which causes the echo pulse electric field to move across the electric field of the fixed LO pulse. Scanning $\tau$ generates an interferogram in $\tau$ for each $\omega_{\mathrm{m}}$. These interferograms are numerically Fourier transformed to give the $\omega_{\tau}$ (horizontal) axis of the 2D spectrum.

A complete $2 \mathrm{D}$ spectrum is generated by fixing $T_{w}$ and scanning $\tau$. Following this, $T_{w}$ is increased and another $2 \mathrm{D}$ spectrum is obtained. The frequency evolution of the vibrational oscillators during the $T_{w}$ period, spectral diffusion, causes the shape of the 2D spectrum to change. Since spectral diffusion is caused by structural evolution of the liquid, the dynamical information of interest is contained in the change in shape of the 2D spectrum as a function of $T_{w}$. When $T_{w}$ is short, relatively few solvent environments have been sampled, resulting in final frequencies that are close to the initial frequencies. The high correlation between the initial and final frequencies at short $T_{w}$ results in a $2 \mathrm{D}$ spectrum that is elongated along the diagonal (line from upper right to lower left corners of the 2D spectrum). As $T_{w}$ is increased, the initial and final frequencies become increasingly uncorrelated. At sufficiently long $T_{w}$, all solvent environments giving rise to the inhomogeneous linewidth of the linear absorption spectrum have been sampled. When all solvent configurations are sampled within the experimental time window, which is limited by the $\mathrm{SeCN}^{-}$vibrational lifetime, the $2 \mathrm{D}$ frequency distribution becomes round, indicating that the initial and final frequencies are uncorrelated.

The 2D IR experiments were performed with linearly polarized electric fields. The signal was acquired in parallel, $\langle X X X X\rangle$, and perpendicular, $\langle X X Y Y\rangle$, polarization configurations. For example, in the $\langle X X Y Y\rangle$ configuration, the first two pulses were vertically polarized $(Y)$ and the third pulse and the echo detection were horizontal $(X)$. The data were acquired using a 4 shot phase cycling scheme discussed in detail previously. ${ }^{32}$ The phase cycling was used to remove scatter signals originating from the pump pulses.

\section{SIMULATION DETAILS}

\section{A. System modeling}

As a first step in the modeling of $\mathrm{SeCN}^{-}$spectroscopy in water, we have developed a fixed charge $\mathrm{SeCN}^{-}$model in concert with the SPC/E model for water $\left(\mathrm{D}_{2} \mathrm{O}\right) .{ }^{33}$ The model is empirical in that the results of the vibrational spectroscopy were used to guide adjustments of the force field parameters, which are given in Table I; Lorentz-Berthelot mixing rules were used for the Lennard-Jones interactions. The $\mathrm{SeCN}^{-}$geometry was optimized at the B3LYP/augcc-pVDZ level with the solvation model based on density (SMD). ${ }^{34}$ The resulting bond distances and bond angle $\left(180^{\circ}\right)$ were taken from this structure. The Lennard-Jones parameters for $\mathrm{C}$ and $\mathrm{N}$ are taken from the ANL model for acetonitrile. ${ }^{35}$ Few models for Se-containing molecules have been reported, so the Lennard-Jones parameters for Se were initially taken as educated guesses to match the expected size of the site and then varied slightly to improve agreement with the measured spectra. Initial atomic charges $\left(q_{\mathrm{Se}}\right.$ $=-0.64097, q_{\mathrm{C}}=0.33471$, and $q_{\mathrm{N}}=-0.69374$ ) were taken from electrostatic potential fitting in the electronic structure calculation mentioned above. However, it was found that smaller absolute charges more accurately reproduced the experimental results, leading to the values given in Table I that were used in the simulations reported here. Specifically, for the ESP-derived charges, the IR linewidth is broader than for the parameters given in Table I $\left(43 \mathrm{~cm}^{-1}\right.$ versus $38.5 \mathrm{~cm}^{-1}$ ); further, increasing the Se Lennard-Jones $\sigma$ from $3.8 \AA$ to $4.0 \AA$ gave slightly faster reorientational and

TABLE I. Force field parameters. $\mathrm{SeCN}^{-}$is linear with $r_{S e-C}=1.82 \AA$ and $r_{C-N}=1.18 \AA ; \mathrm{D}_{2} \mathrm{O}$ has a D-O-D angle of $109.47^{\circ}$ and $r_{O-D}=1.00 \AA$.

\begin{tabular}{lllrr}
\hline \hline Atom & $\sigma(\AA)$ & $\varepsilon(\mathrm{kcal} / \mathrm{mol})$ & $q(\mathrm{e})$ & $m(\mathrm{~g} / \mathrm{mol})$ \\
\hline \multicolumn{5}{c}{$\mathrm{SeCN}^{-}$} \\
\hline $\mathrm{Se}$ & 4.000 & 0.25000 & -0.55 & 78.9600 \\
$\mathrm{C}$ & 3.287 & 0.08365 & 0.10 & 12.0000 \\
$\mathrm{~N}$ & 3.190 & 0.08365 & -0.55 & 14.0067 \\
\hline \multicolumn{5}{c}{$\mathrm{D}_{2} \mathrm{O}$} \\
\hline $\mathrm{O}$ & 3.166 & 0.15535 & -0.8476 & 15.9994 \\
$\mathrm{D}$ & 0.0 & 0.0 & 0.4238 & 2.0158 \\
\hline \hline
\end{tabular}


spectral diffusion dynamics in better agreement with the measurements.

The linear and nonlinear vibrational spectra were obtained by analysis of molecular dynamics simulations that were also used in the generation of an empirical frequency map as described below. The simulation box contains one $\mathrm{SeCN}^{-}$ and $342 \mathrm{D}_{2} \mathrm{O}$ molecules in a cubic cell of side length $21.725311 \AA$. The interactions were calculated with a cutoff of radius $10.5 \AA$ and the long-range electrostatics accounted for with the damped-shifted force method ${ }^{36}$ with a damping parameter of $\alpha=0.2$. The damped-shifted force approach is computationally faster than an Ewald sum and in tests for $\mathrm{SeCN}^{-}$in a $\mathrm{D}_{2} \mathrm{O}$ system gave results for the spectrum and dynamics that were the same within statistical noise. $\mathrm{SeCN}^{-}$ is treated as a rigid molecule, while the $\mathrm{D}_{2} \mathrm{O}$ bonds and angles are fixed using the SHAKE algorithm. ${ }^{37}$ The spectra were calculated from ten 4 ns trajectories. Each trajectory consisted of an initial NVE ensemble equilibration with velocity rescaling to fix the temperature, followed by an equilibration of $0.5 \mathrm{~ns}$ and a data collection stage of $4 \mathrm{~ns}$ in the NVT ensemble. All used a time step of $1 \mathrm{fs}$ and for the $N V T$ stages the temperature was maintained at $298 \mathrm{~K}$ by a Nosé-Hoover thermostat ${ }^{38,39}$ with a thermostat time constant of $100 \mathrm{fs}$. The ten trajectories differed in the length of the velocity rescaling equilibration stage from 0.1 to $1.45 \mathrm{~ns}$, which served to ensure that they are uncorrelated. Error bars were calculated from block averaging (with each of the 10 trajectories as a block) and are reported as $95 \%$ confidence intervals using the Student $t$-distribution. ${ }^{40}$

\section{B. Spectroscopic modeling via an empirical frequency map}

The vibrational spectra of the $\mathrm{CN}$ stretching mode of $\mathrm{SeCN}^{-}$were calculated using an empirical frequency map. In this approach, the vibrational frequencies and transition dipole moments are calculated from a property easily obtained from an MD trajectory, typically an electric field component exerted on the molecule by the solvent. It has been previously observed that this approach fails for alkyl nitriles in various solvents ${ }^{7,41-43}$ (where the frequency shift is very sensitive to charge transfer effects) as well as for anionic solutes in water clusters where more complex approaches have been proposed for $\mathrm{SCN}^{-}{ }^{42,44,45}$ However, we have found that such a relationship can be obtained for $\mathrm{SeCN}^{-}$in aqueous solutions. We calculated the anharmonic vibrational frequency of the $\mathrm{CN}$ stretch in $\mathrm{SeCN}^{-}$in water for 500 configurations sampled every 4 ps from one of the 4 ns MD trajectories described above. The configurations taken from the trajectory are centered at the $\mathrm{SeCN}^{-}$molecule and include the closest $N_{\text {wat }}=9$ water molecules to the anion center-of-mass explicitly included in a density functional theory (DFT) calculation at the B3LYP/augcc-pVDZ level. All additional water molecules within a given cutoff radius of $R_{\text {cut }}=10.0 \AA$ of the $\mathrm{SeCN}^{-}$center-of-mass are included as point charges. The values of $N_{\text {wat }}$ and $R_{c u t}$ were determined from tests on ten configurations where each was varied until the vibrational frequencies and dipole derivative converged.

The vibrational frequency is calculated from the onedimensional anharmonic potential obtained from the DFT calculations, assuming the $\mathrm{CN}$ bond can be treated as a local mode (with a reduced mass of $6.462965 \mathrm{~g} / \mathrm{mol}$ ). Specifically, for each configuration sampled from the MD trajectory, the $\mathrm{CN}$ bond is stretched from 1.00 to $1.48 \AA$ in increments of $0.04 \AA$, keeping the CN center-of-mass fixed, and the DFT energy is calculated for the cluster. These energies are fit to an 8th-order polynomial that is used to generate the potential in a sinc-function discrete variable representation ${ }^{46}$ (DVR) calculation of the vibrational eigenvalues and eigenfunctions. The DVR grid was centered at the potential minimum (taken to be zero of energy) and restricted to $r_{C N} \leq 1.5 \AA$ and $V\left(r_{C N}\right)$ $\leq 2.3 \mathrm{eV}$ with a grid spacing of $0.00497 \AA$; these parameters give grids with 84-92 DVR points.

The vibrational frequencies $\omega_{01}$ and $\omega_{12}$ as well as the transition dipole moment derivative absolute value, $\left|\mu^{\prime}\right|$, are shown in Fig. 1 (black points) as a function of the electric field component along the $\mathrm{SeCN}^{-}$molecular axis evaluated at the carbon atom, $\varepsilon_{C}$. There is a clear linear correlation between both of these properties and the electric field $\varepsilon_{C}$ (Fig. 1, dashed red lines); the empirical map relationships are given in Table II.
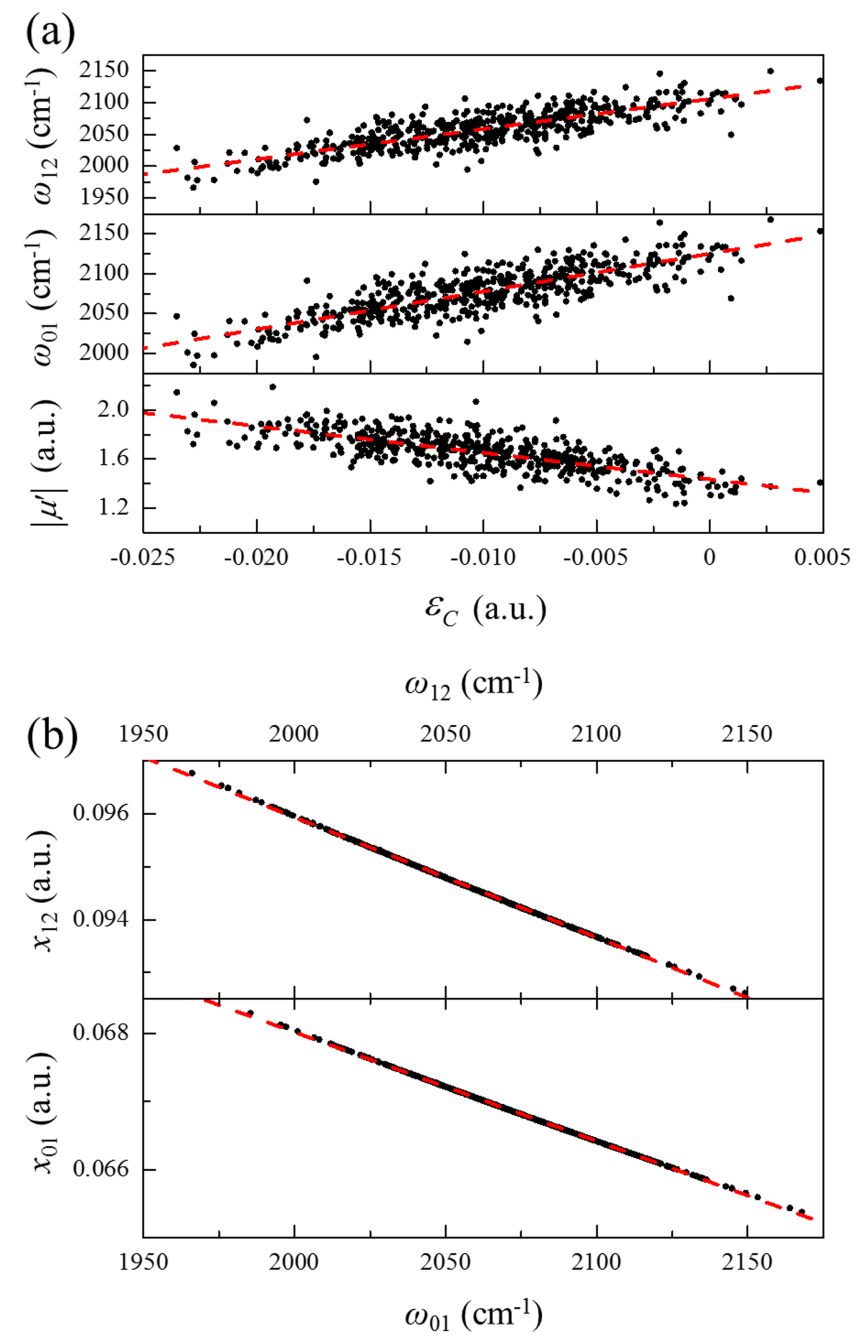

FIG. 1. (a) The DFT-calculated fundamental $\mathrm{CN}$ stretching frequencies $\omega_{01}$ (middle) and $\omega_{12}$ (top) and the absolute value of the transition dipole derivative, $\left|\mu^{\prime}\right|$ (bottom), are plotted versus the electric field along the $\mathrm{SeCN}^{-}$ molecule evaluated at the $\mathrm{C}$ atom, $\varepsilon_{C}$ (filled black circles). (b) The $x_{01}$ (bottom) and $x_{12}$ (top) matrix elements are plotted versus the $\omega_{01}$ and $\omega_{12}$ frequencies, respectively (filled black circles). In all cases, the empirical map fits are also shown (dashed red lines). 
TABLE II. Empirical map parameters. Frequencies, $\omega_{01}$ and $\omega_{12}$, are in $\mathrm{cm}^{-1}$ while all remaining quantities are in atomic units.

\begin{tabular}{lc}
\hline \hline Map equation & RMSD \\
\hline$\omega_{01}=2125.186+4748.241 \varepsilon_{C}$ & 17.4 \\
$\omega_{12}=2105.775+4738.519 \varepsilon_{C}$ & 17.5 \\
$x_{01}=0.099963-1.597401 \times 10^{-5} \omega_{01}$ & $7.4 \times 10^{-6}$ \\
$x_{12}=0.141308-2.268885 \times 10^{-5} \omega_{12}$ & $1.2 \times 10^{-5}$ \\
$\mu^{\prime}=-1.43293+21.7139487 \varepsilon_{C}$ & 0.11 \\
\hline \hline
\end{tabular}

It is important to note that this map, while relating the frequencies to an electric field, is not representative of a Stark effect but instead is distinctly empirical. This is clearly demonstrated by the fact that there is no clear correlation between the frequency and the field evaluated at the nitrogen atom of $\mathrm{SeCN}^{-}$and hence between the frequency and the electric field exerted on the $\mathrm{CN}$ bond. We have constructed empirical maps based on three variations of the $\mathrm{SeCN}^{-}$force field parameters and all three gave linear correlations between the spectroscopic quantities (frequencies and dipole derivative) and $\varepsilon_{C}$. Table II and Fig. 1 present the map and force field that give the best agreement with the experimental spectroscopic measurements.

The linear IR spectrum is calculated within the empirical mapping framework as the Fourier transform,

$$
I(\omega)=\frac{1}{2 \pi} \int_{-\infty}^{\infty} e^{-i \omega t} \phi(t) d t,
$$

of the dipole-dipole response function,

$$
\phi(t)=\left\langle\vec{\mu}_{01}(0) \cdot \vec{\mu}_{01}(t) e^{i \int_{0}^{t} \omega(\tau) d \tau}\right\rangle e^{|t| / 2 T_{1}},
$$

where $\omega(t)$ and $\vec{\mu}_{01}(t)$ are the instantaneous $0 \rightarrow 1$ frequency and transition dipole moment at time $t$, which are obtained from the empirical map (the latter using $\vec{\mu}_{01}=\mu^{\prime} x_{01} \vec{e}$, where $\vec{e}$ is a unit vector along the $\mathrm{CN}$ bond and $x_{n m}$ is the matrix element of the stretching coordinate between vibrational states $n$ and $m$ determined by the empirical map relations in Table II). The vibrational energy relaxation time, $T_{1}=36.2 \mathrm{ps}$, is taken from the measurements in this work. The 2D IR spectra are calculated according to the approach described in Ref. 47 using the third-order nonlinear response functions, which involve both the $0 \rightarrow 1$ and $1 \rightarrow 2$ transition frequencies and dipole moments obtained from the empirical map given in Table II. The simulations correspond to the measured isotropic 2D IR spectra.

\section{RESULTS AND DISCUSSION}

\section{A. Linear IR spectrum}

Figure 2(a) shows the measured linear absorption spectrum of the $\mathrm{CN}$ stretch of $\mathrm{SeCN}^{-}$in $\mathrm{D}_{2} \mathrm{O}$ (black curve). The shape of the experimental spectrum is identical for 0.1 and $0.3 \mathrm{M}$ solutions, with a $2074.7 \pm 0.1 \mathrm{~cm}^{-1}$ center frequency and $32.9 \pm 0.1 \mathrm{~cm}^{-1}$ FWHM. Fitting a Voigt line shape function (red curve) to the blue (higher frequency) side and peak of the measured spectrum and extending the fit across the entire frequency range clearly shows that the spectrum is

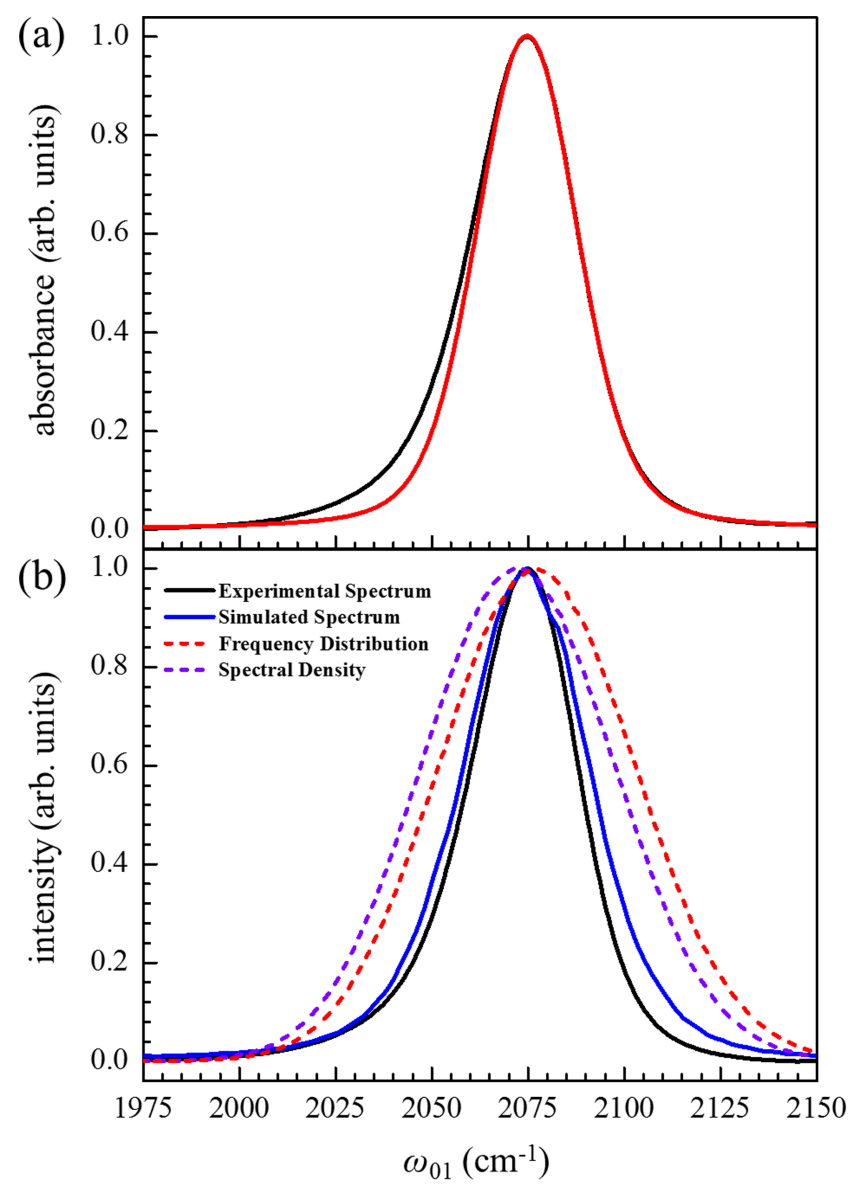

FIG. 2. (a) Background-subtracted linear IR spectrum of the $\mathrm{CN}$ stretch of $\mathrm{SeCN}^{-}$in $\mathrm{D}_{2} \mathrm{O}$ (black curve). A Voigt fit (red curve) to the blue side and peak of the spectrum is extended across the full frequency range, showing that the absorption spectrum is not symmetric. The spectrum has a red side wing that is shown to arise from the non-Condon effect. (b) Simulated IR spectrum (solid blue curve) of the $\mathrm{CN}$ stretch of $\mathrm{SeCN}^{-}$in $\mathrm{D}_{2} \mathrm{O}$ compared to the measured spectrum (black curve), the $\omega_{01}$ vibrational frequency distribution, $P(\omega)=\left\langle\delta\left(\omega-\omega_{01}\right)\right\rangle$ (dashed red curve), and the spectral density, $P_{\mu}(\omega)=\left\langle\left|\mu_{01}\right|^{2} \delta\left(\omega-\omega_{01}\right)\right\rangle$ (dashed violet curve).

asymmetric with excess absorption on the red (lower frequency) side. Similar red wings have been observed for HOD in $\mathrm{D}_{2} \mathrm{O},{ }^{1}$ as well as for water and linear alcohols forming hydrogen bonds in the charged solvation structures of alkyl imidazolium based room temperature ionic liquids (RTILs). ${ }^{48}$ However, no red wing is observed when $\mathrm{SeCN}^{-} / \mathrm{D}_{2} \mathrm{O}$ solutions are confined in $w_{0}=1$ AOT reverse micelles, in which hydrogen bonding between $\mathrm{SeCN}^{-}$and $\mathrm{D}_{2} \mathrm{O}$ is statistically unlikely due to the low water content of the micelles. These results suggest that the absence of the red wing in reverse micelles is caused by a lack of hydrogen bonding of water to the $\mathrm{CN}$ moiety. ${ }^{49}$ Thus, the red wing appears to originate from an asymmetry related to the hydrogen bonding interactions between $\mathrm{SeCN}^{-}$and $\mathrm{D}_{2} \mathrm{O}$.

The simulated line shape (blue curve) is compared to the measured spectrum (black curve) in Fig. 2(b). The simulated peak position of $2075 \mathrm{~cm}^{-1}$, obtained without any frequency scaling, is in excellent agreement with the experimental spectrum, while the linewidth of $38.5 \mathrm{~cm}^{-1}$ is only slightly broader than the measured one. We note that, in addition to being broader, the simulated spectrum does not exhibit 
the same asymmetry associated with increased absorption on the red side of the spectrum as observed in the measurements. For comparison, the distribution of frequencies, $P(\omega)=\langle\delta(\omega$ $\left.\left.-\omega_{01}\right)\right\rangle$, is also shown (Fig. 2(b), red dashed curve). It is slightly blue shifted, by $\sim 2 \mathrm{~cm}^{-1}$, and is significantly broadened relative to the simulated spectrum, with $59.6 \mathrm{~cm}^{-1}$ FWHM. The broader frequency distribution compared to the IR spectrum suggests an important role for non-Condon (frequency dependent transition dipole) and/or motional narrowing effects in determining the line shape.

Since the linear absorption at a given frequency depends on the population and the second power of the transition dipole moment at that frequency, ${ }^{11}$ an excess of solvent structures giving rise to red frequencies or enhanced transition dipole strength on the red side of the line relative to the blue side can generate a red wing. Simulations ${ }^{41-43}$ and experiments ${ }^{49,50}$ have found that the transition dipole of nitriles in hydrogen bonding solvents is frequency dependent, i.e., the non-Condon effect. Indeed, this is reflected in the DFT-derived data for the frequency and transition dipole derivative shown in Fig. 1(a) and the corresponding empirical map formulas given in Table II. Specifically, the simulations indicate that as the frequency decreases, the magnitude of the transition dipole moment, $\left|\mu_{01}\right|=\left|\mu^{\prime} x_{01}\right|$, increases. The change is modest, giving an increase in intensity of $\sim 1.2$ from the blue to red side of FWHM. This effect is represented in the spectral density, $P_{\mu}(\omega)=\left\langle\left|\mu_{01}\right|^{2} \delta\left(\omega-\omega_{01}\right)\right\rangle$, that weights the frequency distribution by the strength of the transition dipole moment, and is compared (dashed violet curve) to the simulated spectrum and $P(\omega)$ in Fig. 2(b). The non-Condon effects lead to a spectral density that is shifted to the red side by $\sim 5 \mathrm{~cm}^{-1}$ compared to the frequency distribution. However, the FWHM of $58.8 \mathrm{~cm}^{-1}$ is only slightly smaller than that of $P(\omega)$, indicating that the IR linewidth is not primarily determined by non-Condon effects but rather motional narrowing that produces the homogeneous linewidth. The absence of the asymmetry in the simulated spectrum may indicate that the model under-represents the redshifted configurations. Alternatively, the red wing may result from reduced motional narrowing at lower frequencies that is not properly described in the simulations.

A comparison of the $\mathrm{CN}$ stretch of $\mathrm{SeCN}^{-}$in the polar aprotic solvent dimethylformamide (DMF) versus the polar protic solvent formamide (FA) found an increase in the peak frequency $\left(2066.5 \mathrm{~cm}^{-1}\right.$ vs. $\left.2068 \mathrm{~cm}^{-1}\right)$ and linewidth $\left(12.5 \mathrm{~cm}^{-1}\right.$ vs. $\left.24.0 \mathrm{~cm}^{-1}\right)$, respectively. ${ }^{50}$ This suggests that $\mathrm{SeCN}^{-}$oscillators involved in stronger hydrogen bonds are shifted to the blue side. ${ }^{49}$ This is supported by gas phase harmonic frequency calculations at the B3LYP/aug-cc-pVDZ level of a single $\mathrm{SeCN}^{-}$and a $\mathrm{SeCN}^{-} \cdots \mathrm{H}_{2} \mathrm{O}$ complex. These show a blue shift of $\sim 11 \mathrm{~cm}^{-1}$ in the $\mathrm{CN}$ stretch frequency upon hydrogen bonding as well as an increase in the IR intensity by roughly a factor of 1.6. These effects have been observed for other nitriles ${ }^{42,43,51}$ and attributed to charge transfer from the nitrogen lone pair to the hydroxyl $\sigma^{*}$ orbital. The lone pair has some $\mathrm{CN}$ bond $\pi^{*}$ character. Charge transfer out of the lone pair leads to a strengthening of the bond and increases the transition dipole moment. The frequency blueshift can equivalently be explained in terms of changes in the contributions of the two dominant $\mathrm{SeCN}^{-}$resonance structures, $[\mathrm{Se}-\mathrm{C} \equiv \mathrm{N}]^{-}$and $[\mathrm{Se}=\mathrm{C}=\mathrm{N}]^{-} .{ }^{49}$ In contrast to $\mathrm{SeCN}^{-}$, water hydroxyls forming stronger hydrogen bonds are shifted to the red. ${ }^{52}$ However, the two systems are similar in that the transition dipole strength is found to be stronger on the red side of the line in both cases. It is also worth emphasizing that the hydrogen bonding interactions between $\mathrm{SeCN}^{-}$and $\mathrm{D}_{2} \mathrm{O}$ are relatively strong and broadly distributed relative to other liquids. As a reference, the peak position is $\sim 11 \mathrm{~cm}^{-1}$ higher and the width is $\sim 11 \mathrm{~cm}^{-1}$ wider than when the anion is dissolved in the RTIL 1-ethyl-3-methylimidazolium bis(trifluoromethylsulfonyl)imide $\left(\right.$ EmimNTf $\left._{2}\right) .53$

These relationships between hydrogen bonding and both the $\mathrm{CN}$ frequency and transition dipole moment were investigated in the simulations. Hydrogen bonds between $\mathrm{D}_{2} \mathrm{O}$ and $\mathrm{SeCN}^{-}$were defined by geometric criteria based on the $\mathrm{O} \cdots \mathrm{N}$ and D $\cdots$ N distances, $R_{O N} \leq 3.5 \AA$ and $r_{D N} \leq 2.45 \AA$, respectively, and the $\mathrm{D} \cdots \mathrm{N} \cdots \mathrm{O}$ angle, $\theta_{D N O} \leq 30^{\circ}$. The probabilities of different total numbers of hydrogen bonds are shown in Fig. 3. On average, there are 3.4 hydrogen bonds to $\mathrm{SeCN}^{-}$ from $\mathrm{D}_{2} \mathrm{O}$ molecules.

The effect of the hydrogen bonding environment on the spectra can be examined by decomposing the spectral density, $P_{\mu}(\omega)$, according to the number of hydrogen bonds present between $\mathrm{D}_{2} \mathrm{O}$ and $\mathrm{SeCN}^{-}$for each configuration in the MD simulations. This is shown in Fig. 4. The results clearly indicate that the $\mathrm{CN}$ stretching frequency shifts to the red side as the number of hydrogen bonds increases, with average frequencies of $2124,2105,2083,2066$, and $2054 \mathrm{~cm}^{-1}$ for $N_{\mathrm{HB}}=$ $1-5$, respectively, as shown in Fig. 3 (black points). The spectral density distributions for the different $N_{H B}$ values are broad and overlapping, but are well described as Gaussians with FWHM values of $48.9,49.3,50.6,52.2$, and $54.0 \mathrm{~cm}^{-1}$, for $N_{H B}=1-5$, respectively, all narrower than $58.8 \mathrm{~cm}^{-1}$ of the total spectral density (Fig. 4, black curve). These results, together with those presented above, indicate that the IR linewidth is a combination of both inhomogeneous broadening due to the variation in hydrogen bonding arrangements and motional narrowing.

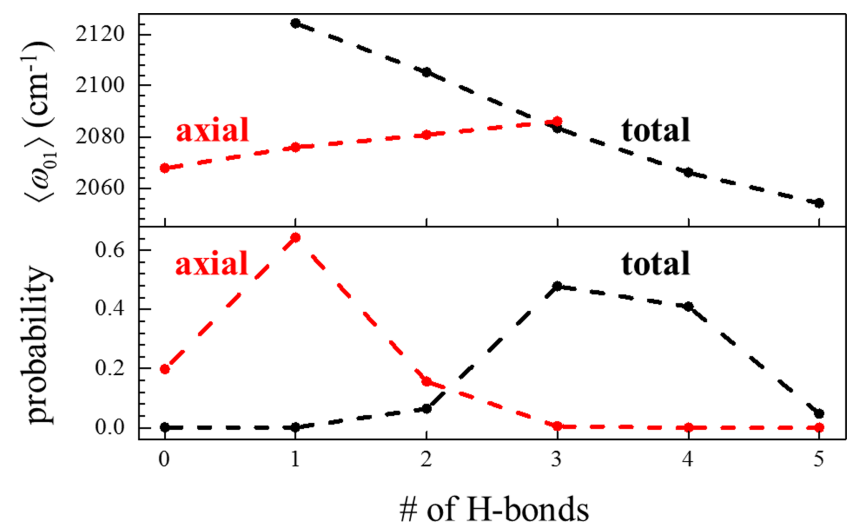

FIG. 3. Bottom: calculated probability of the total number of hydrogen bonds donated to $\mathrm{SeCN}^{-}$by $\mathrm{D}_{2} \mathrm{O}$ (black circles) and the number of axial hydrogen bonds (red circles) defined as $\theta_{\mathrm{CND}} \geq 120^{\circ}$. Top: average fundamental transition frequency, $\left\langle\omega_{01}\right\rangle$, as a function of the total number of hydrogen bonds (black circles) and number of axial hydrogen bonds (red circles). Dashed lines are shown as a guide to the eye. 


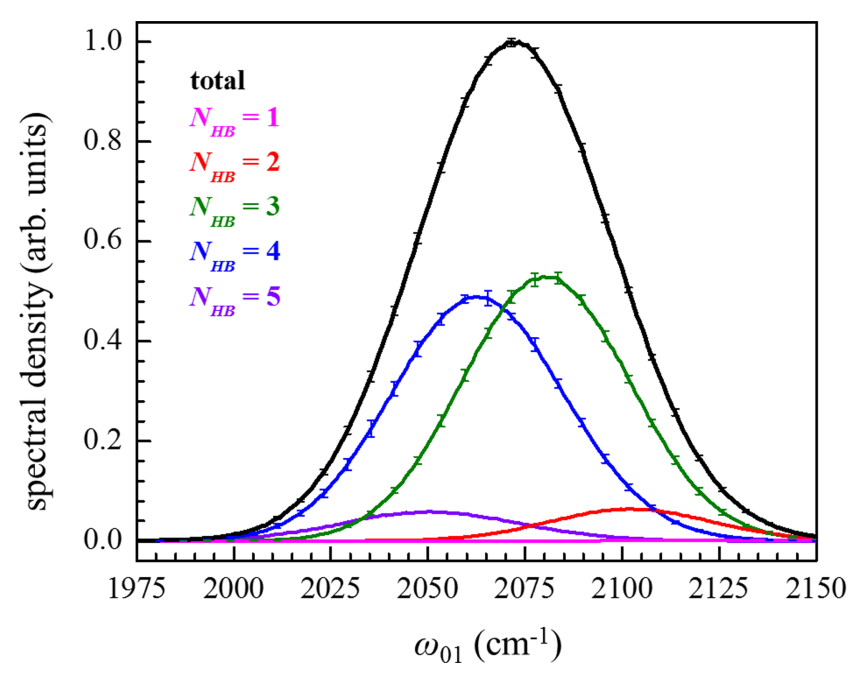

FIG. 4. Spectral density decomposed according to the total number of hydrogen bonds donated to $\mathrm{SeCN}^{-}$by $\mathrm{D}_{2} \mathrm{O}$. Results are shown for the total (black curve) and $N_{H B}=1$ (magenta), 2 (red), 3 (green), 4 (blue), and 5 (violet).

The large number of hydrogen bonds to $\mathrm{SeCN}^{-}$and the red shift with increasing $N_{H B}$ deserve further examination. The latter is particularly surprising in light of the well-known blue shift induced in nitrile stretching frequencies upon hydrogen bonding. ${ }^{10}$ Additional insight can be obtained by further characterizing the hydrogen bonds as "axial," defined as those for which the $\mathrm{C} \cdots \mathrm{N} \cdots \mathrm{D}$ angle, $\theta_{C N D}$, is greater than $120^{\circ}$. In this way, the hydrogen bond donors that are pointing more nearly parallel to the $\mathrm{SeCN}^{-}$axis can be distinguished from those that are equatorial, i.e., more perpendicular to the $\mathrm{CN}$ bond. The distribution of these axial hydrogen bonds is shown in Fig. 3 (lower panel, red points) and the average number is 0.97 . Thus, the analysis shows that there are nearly always multiple hydrogen bonds including one axial hydrogen bond, but also additional weaker, equatorial ones. Calculation of the average frequency shows a blue shift with an increasing number of axial hydrogen bonds as shown in Fig. 3 (top panel, red points). The dependence of the average frequency on the number of axial hydrogen bonds is weaker than that for the total number of hydrogen bonds, varying as 2068,2076 , and $2080 \mathrm{~cm}^{-1}$ for 0,1 , and 2 axial donors, respectively.

Taken together, these results indicate that the $\mathrm{CN}$ stretch frequency is blue shifted overall by the presence of a strong, axial hydrogen bond, but additional, weaker equatorial hydrogen bonds shift the frequency back to the red side. This is consistent with calculations of the $\mathrm{CN}$ stretching frequency in acetonitrile-water dimers that show blue shifts for axial arrangements and redshifts for equatorial configurations. ${ }^{54}$ The additional hydrogen bonds also increase the transition dipole moment, leading to the non-Condon effect favoring the redshifted frequencies.

\section{B. Isotropic pump-probe decays}

In the PSPP experiments, the pump-probe signals parallel, $S_{\|}(t)$, and perpendicular, $S_{\perp}(t)$, to the pump polarization $\left(45^{\circ}\right)$ are measured. The time dependence of the measured signals can be decomposed into contributions from the isotropic pump-probe decay, $P(t)$, and the second order Legendre polynomial orientational correlation function of the transition dipole moment, $C_{2}(t),{ }^{55}$

$$
\begin{aligned}
& S_{\|}(t)=P(t)\left[1+0.8 C_{2}(t)\right], \\
& S_{\perp}(t)=P(t)\left[1-0.4 C_{2}(t)\right] .
\end{aligned}
$$

$C_{2}(t)$ reports on the orientational dynamics of the transition dipole direction ${ }^{30}$ which, for the $\mathrm{CN}$ stretch of $\mathrm{SeCN}^{-}$, is parallel to the $\mathrm{CN}$ bond vector. The correlation function $C_{2}(t)$ is given by

$$
C_{2}(t)=\left\langle P_{2}[\vec{e}(t) \cdot \vec{e}(0)]\right\rangle,
$$

where $P_{2}$ is the second-order Legendre polynomial and $\vec{e}(t)$ is the transition dipole moment unit vector at time $t .\langle\ldots\rangle$ represents an isotropic ensemble average.

$P(t)$ is independent of orientation and reports on the population relaxation of the excited vibration; however, it may also be influenced by additional isotropic dynamics. ${ }^{48}$ The isotropic pump-probe signal is a linear combination of the parallel and perpendicular signals,

$$
P(t)=\left[S_{\|}(t)+2 S_{\perp}(t)\right] / 3 .
$$

Three $P(t)$ curves at red, center, and blue locations in the absorption spectrum of $\mathrm{SeCN}^{-}$in $\mathrm{D}_{2} \mathrm{O}$ are displayed in Fig. 5 . At very short times, an ultrafast non-resonant signal on the order of the pulse durations obscures the dynamics. To avoid the non-resonant artifact, the data (points) begin at a delay of $0.5 \mathrm{ps}$ and extend to $200 \mathrm{ps}$. A small offset, persisting at very long time, was attributed to isotropic heating arising from vibrational relaxation. The data were fit with an offset to remove the heating signal from the analysis. This procedure gave identical numbers to the more involved procedure previously reported. ${ }^{3,56}$ As shown in the inset of Fig. 5, which expands the early time portion of the curves, the decays are not single exponential. In addition to a slow exponential decay,

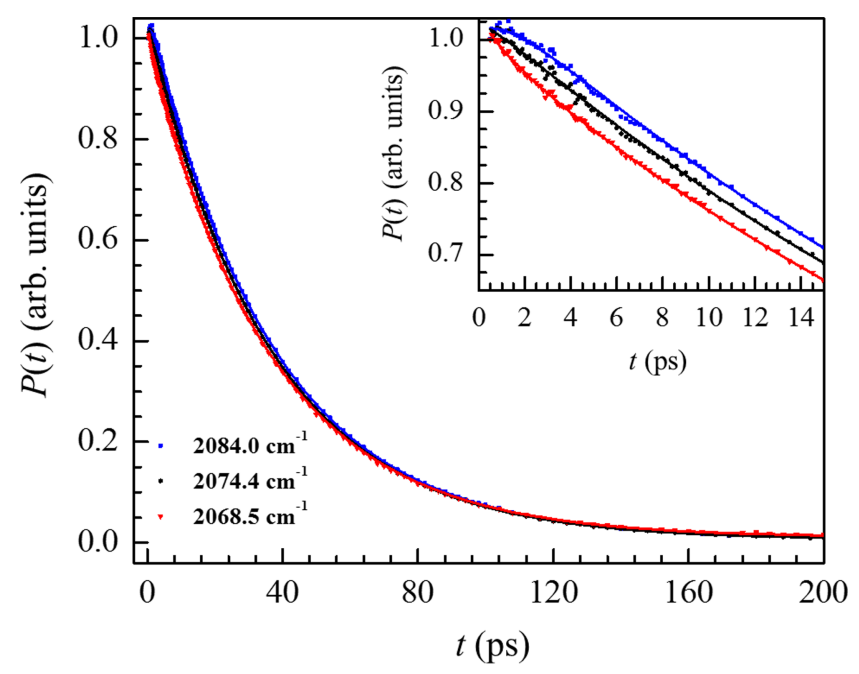

FIG. 5. Isotropic pump-probe decays at three frequencies across the absorption spectrum of $\mathrm{SeCN}^{-}$in $\mathrm{D}_{2} \mathrm{O}$. The data (points) are normalized to the value at the first time point, $0.5 \mathrm{ps}$. The solid curves are biexponential fits to the data. The inset displays the early time portion of the curves. A fast exponential decay and growth, occurring on a $1.4 \mathrm{ps}$ time scale, is observed at red $\left(2068.5 \mathrm{~cm}^{-1}\right)$ and blue $\left(2084.0 \mathrm{~cm}^{-1}\right)$ frequencies, respectively. The $1.4 \mathrm{ps}$ feature is a result of spectral diffusion. The slower, frequency-independent decay time of $36.2 \pm 0.1 \mathrm{ps}$ is the $\mathrm{SeCN}^{-}$vibrational lifetime. 
on the red side of the spectrum $\left(2068.5 \mathrm{~cm}^{-1}\right)$, a fast decay is observed, while on the blue side $\left(2084.0 \mathrm{~cm}^{-1}\right)$, a fast growth is seen. Near the center frequency $\left(2074.4 \mathrm{~cm}^{-1}\right)$, the fast process essentially vanishes. The progression from rapid decay to rapid growth in the first 4-5 ps of the isotropic pump-probe signal is observed across all frequencies moving from red to blue.

When the $P(t)$ curves are fit starting at $t>10 \mathrm{ps,}$ the data decay as frequency independent single exponentials with a time constant of 36.2 ps. To fit the multiple data sets taken at different frequencies, the short and long time constants were shared among frequencies in the range 2068.5-2094.0 $\mathrm{cm}^{-1}$ in the biexponential fits to the data, but the amplitudes of the two components were allowed to vary. Using this fitting procedure, a value of $1.4 \pm 0.1$ ps was obtained for the fast time scale component. At frequencies red of the line center, the amplitude of this component is positive, corresponding to a fast decay. Moving to blue frequencies, the amplitude switches sign (negative), corresponding to a growth. Similar features in the isotropic pump-probe signal have been observed before, ${ }^{48,49}$ and in the case of $\mathrm{SeCN}^{-}$in $\mathrm{D}_{2} \mathrm{O}$, are indicative of population transfer across the absorption line from red to blue frequencies by means of spectral diffusion. As discussed below, the 2D IR experiments give $1.4 \mathrm{ps}$ as the time constant of the dominant component of the spectral diffusion. The net flow of population from red to blue occurs because a non-equilibrium excited state distribution with excess population at lower frequencies was prepared by the pump pulse. However, the pump spectrum was tuned to the center of the absorption spectrum and had a much larger bandwidth $\left(\sim 110 \mathrm{~cm}^{-1}\right.$ vs. $\sim 33 \mathrm{~cm}^{-1}$ FWHM). Given the essentially uniform intensity of the excitation pulse over the absorption spectrum, unequal pumping of the absorption line cannot explain the observed non-equilibrium distribution of excited states.

As discussed above, the red wing of the absorption spectrum [see Fig. 2(a)] is caused, at least in part, by the nonCondon effect, with molecules on the red side having larger transition dipoles. Consequently, despite uniform pumping intensity across the band, more absorption occurs on the red side of the line than dictated by the ground state thermal equilibrium distribution, resulting in a non-equilibrium excited state distribution. Spectral diffusion, the process by which oscillators sample different frequencies via structural fluctuations of the sample medium, is the mechanism by which the initial non-equilibrium excited population distribution evolves to the thermal equilibrium distribution. Population on the overpumped red side of the line moves to the blue, causing a fast decay on the red side and a build up with the same time constant on the blue side of the line. The fast component of the isotropic decay $(1.4 \pm 0.1 \mathrm{ps})$ is identical to the slowest component of the spectral diffusion measured $(1.4 \pm 0.2 \mathrm{ps})$. The slower, frequency independent, component of the isotropic decay, $36.2 \pm 0.1 \mathrm{ps}$, is the vibrational lifetime. Therefore, $\mathrm{SeCN}^{-}$in $\mathrm{D}_{2} \mathrm{O}$ is a single population that decays with a uniform vibrational lifetime across the band but displays the influence of spectral diffusion at short times because of the non-Condon effect.

The present simulations based on the empirical mapping approach for describing $\mathrm{SeCN}^{-}$spectroscopy do not provide information on the vibrational relaxation of the $\mathrm{CN}$ stretching mode. This will be the topic of a future study.

\section{Orientational relaxation}

The orientational relaxation dynamics of $\mathrm{SeCN}^{-}$, which are contained in the correlation function $C_{2}(t)$, were extracted from the PSPP data by calculating the anisotropy, ${ }^{55}$

$$
r(t)=\frac{S_{\|}(t)-S_{\perp}(t)}{S_{\|}(t)+2 S_{\perp}(t)}=0.4 C_{2}(t) .
$$

Figure 6(a) displays $r(t)$ at four frequencies across the range 2068.0-2084.7 $\mathrm{cm}^{-1}$. The data (points) and fits (solid curves, see below) are plotted for $t=0.5-30 \mathrm{ps}$. In addition to those shown in Fig. 6(a), the anisotropy decays appear essentially identical at all frequencies across the band. The $r(t)$ are well described by a biexponential decay. The insets show the residuals for a single exponential fit (top) and a biexponential fit (bottom). Although the curves could be fit very well with no offset, an independent offset was included to avoid the introduction of a small systematic error into the parameters of the fits. The average offset was $\sim 0.1 \%$ of the



FIG. 6. (a) The anisotropy of $\mathrm{SeCN}^{-}$in $\mathrm{D}_{2} \mathrm{O}$ at four frequencies across the range 2068.0-2084.7 $\mathrm{cm}^{-1}$. The data (points) are fit with a wobbling-in-a-cone model (solid curves). The top and bottom insets display the residuals obtained with single and biexponential fits to the data, respectively. (b) The simulated orientational correlation function, $0.4 C_{2}(t)$, (blue curve) and the measured anisotropy, $r(t)$, (black circles) of $\mathrm{SeCN}^{-}$in $\mathrm{D}_{2} \mathrm{O}$. 
total amplitude. The amplitudes and time constants of the biexponential fits were shared across all frequencies. The fit parameters at the center of the band are displayed in Table III. We note that the orientational correlation function is independent of $P(t)$. Consequently, $r(t)$ does not reflect the vibrational lifetime or spectral diffusion dynamics observed in $P(t)$.

The orientational correlation function, $C_{2}(t)$, was also calculated from the MD simulation data. The results, presented as $0.4 C_{2}(t)$ [Fig. 6(b), blue curve], are compared to the measured anisotropy at the band center, $r(t)$ [Fig. 6(b), black points], and the parameters obtained from a biexponential fit are given in Table III. The agreement is excellent. Note that the orientational dynamics are obtained without reference to the empirical map and depend only on the force field parameters.

Previous studies on the reorientation of dilute $(\leq 0.5 \mathrm{M})$ $\mathrm{KSCN}^{19,20}$ and $\mathrm{KSeCN}^{49}$ in bulk water reported single exponential fits to the anisotropy. The points in Fig. 6 are the mean of 3 separate PSPP experiments on $0.3 \mathrm{M} \mathrm{KSeCN}$ in $\mathrm{D}_{2} \mathrm{O}$. The residuals obtained from single exponential fits (amplitudes and time constants shared, independent offsets) to the data are displayed in the top inset of Fig. 6(a). A clear trend in the residuals is apparent. The fits initially systematically fall below the data, then above the data at intermediate times, and then below again at long time. In contrast, the biexponential fits to the data yield residuals [Fig. 6(a), bottom inset] that are centered about zero. The biexponential fits were also preferred over the single exponential fits when subjected to the Akaike Information Criterion (AIC). ${ }^{57}$ The observation of two time scales here may be the result of improved signal to noise and reduced pulse durations (improved temporal resolution) relative to previous studies.

The biexponential form of the anisotropy decays reflects the orientational relaxation dynamics of a single population of absorbers (see Sec. IV B). Therefore, we interpret the smaller amplitude time scale, $t_{1}=1.4 \mathrm{ps}$, as restricted orientational diffusion of $\mathrm{SeCN}^{-}$, which occurs on a faster time scale than the process of complete orientational randomization. The simulations predict a slightly faster time scale of $t_{1}=0.9 \mathrm{ps}$ for this motion. The measured biexponential anisotropy decays were analyzed with the wobbling-in-a-cone model $^{58-60}$ of restricted orientational diffusion. The model assumes that the transition dipole freely diffuses within a restricted volume of angular space defined by a cone of half angle $\theta_{c}$. The orientational correlation function for a single diffusive cone is given by

$$
C_{2}(t)=Q^{2}+\left(1-Q^{2}\right) \exp \left[-t / \tau_{c}\right]
$$

where $\tau_{c}$ is the correlation time for restricted angular diffusion and $Q^{2}$ is the square of a generalized order parameter,

$$
Q^{2}=\left[\frac{1}{2} \cos \theta_{c}\left(1+\cos \theta_{c}\right)\right]^{2} \text {. }
$$

In the long time limit, $C_{2}(t)$ decays to a plateau with the value $Q^{2}$. Greater restrictions on orientational diffusion result in larger values of $Q^{2}$ and smaller cone angles, $\theta_{c}$. The orientational diffusion constant for wobbling-in-a-cone of half angle $\theta_{c}$, order parameter $Q$, and correlation time $\tau_{c}$ is given by 59

$$
\begin{aligned}
D_{c}= & \frac{x_{c}^{2}\left(1+x_{c}\right)^{2}\left\{\ln \left[\left(1+x_{c}\right) / 2\right]+\left(1-x_{c}\right) / 2\right\}}{\tau_{c}\left(1-Q^{2}\right)\left[2\left(x_{c}-1\right)\right]} \\
& +\frac{\left(1-x_{c}\right)\left(6+8 x_{c}-x_{c}^{2}-12 x_{c}^{3}-7 x_{c}^{4}\right)}{24 \tau_{c}\left(1-Q^{2}\right)}
\end{aligned}
$$

where $x_{c}=\cos \theta_{c}$.

The diffusive cone orientational correlation function [Eq. (8)] must be modified to fit the full extent of the anisotropy decay. First, owing to ultrafast inertial angular motion of the probe anion, the value of $r(t)$ at $t=0, \sim 0.38$, falls below the theoretical value of 0.4 [Eq. (7)]. The pulse durations used in the experiments are too long to resolve the limited inertial angular motion, which occurs on a time scale $<100$ fs. ${ }^{1,2}$ The inertial dynamics are included in the simulated orientational correlation function but, consistent with the measurements, they are small in magnitude as can be seen from the short-time behavior of $C_{2}(t)$ [Fig. 6(b), blue curve]. Although the inertial motion is not diffusive in nature, it can still be accounted for with $C_{2}(t)=T^{2}$, where $T$ is the generalized order parameter for the inertial reorientation. This expression is obtained from Eq. (8) in the limit that $\tau_{c}$ goes to zero. We will denote the diffusive cone order parameter as $S$ to distinguish it from the inertial cone order parameter, $T$. Second, the complete long time diffusive orientational randomization has the form $^{30,55}$

$$
C_{2}(t)=\exp \left[-6 D_{m} t\right]
$$

where $D_{m}=1 / 6 \tau_{m}$ is the orientational diffusion constant and $\tau_{m}$ is the correlation time for free diffusion. We note that one check on the appropriateness of the Debye model is its prediction that $\tau_{1} / \tau_{2}=3$, where $\tau_{1}$ and $\tau_{2}$ are the time scales for the decay of the $C_{1}(t)$ and $C_{2}(t)$ orientational correlation functions; the simulations give this ratio as 2.6 (2.8 if the correlation functions are fit by single exponentials), in good agreement with the model. The full expression for the total orientational correlation function as a product of the individual functions is given by ${ }^{61}$

$$
C_{2}(t)=T^{2}\left(S^{2}+\left(1-S^{2}\right) \exp \left[-t / \tau_{c}\right]\right) \exp \left[-t / \tau_{m}\right] .
$$

TABLE III. Anisotropy fit parameters. $A_{i}$ and $t_{i}$ are the amplitude and time constant of the $i$ th component of the biexponential fit to the anisotropy data, respectively. The amplitudes and time constants were shared across the band. $y_{0}$ is an independent offset.

\begin{tabular}{lccccc}
\hline \hline & $A_{1}$ & $t_{1}(\mathrm{ps})$ & $A_{2}$ & $t_{2}(\mathrm{ps})$ & $y_{0}$ \\
\hline Experiment $\left(2075.1 \mathrm{~cm}^{-1}\right)$ & $0.073 \pm 0.002$ & $1.4 \pm 0.1$ & $0.304 \pm 0.003$ & $4.5 \pm 0.1$ & $-0.0011 \pm 0.0002$ \\
Simulation & $0.10 \pm 0.01$ & $0.9 \pm 0.1$ & $0.29 \pm 0.01$ & $4.5 \pm 0.1$ & 0 \\
\hline \hline
\end{tabular}


TABLE IV. Wobbling-in-a-cone parameters. See text for parameter descriptions.

\begin{tabular}{lccccccc}
\hline \hline & $\theta_{\text {in }}\left({ }^{\circ}\right)$ & $\theta_{c}\left({ }^{\circ}\right)$ & $\theta_{\text {tot }}\left({ }^{\circ}\right)$ & $\tau_{c}(\mathrm{ps})$ & $\tau_{m}(\mathrm{ps})$ & $D_{c}\left(\mathrm{ps}^{-1}\right)$ & $D_{m}\left(\mathrm{ps}^{-1}\right)$ \\
\hline Experiment $\left(2075.1 \mathrm{~cm}^{-1}\right)$ & $11.3 \pm 0.1$ & $21.5 \pm 0.4$ & $24.2 \pm 0.3$ & $2.0 \pm 0.1$ & $4.5 \pm 0.1$ & $0.020 \pm 0.001$ & $0.0370 \pm 0.0002$ \\
Simulation & $4.0 \pm 0.6$ & $25.4 \pm 0.2$ & $25.7 \pm 0.2$ & $1.1 \pm 0.1$ & $4.5 \pm 0.1$ & $0.047 \pm 0.002$ & $0.0371 \pm 0.0001$ \\
\hline \hline
\end{tabular}

The anisotropy decays at all frequencies were simultaneously fit to the expression obtained from substituting Eq. (12) into Eq. (7) [Fig. 6(a), solid curves]. A single set of order parameters and correlation times were used in the fit. As in the biexponential fits, a small independent offset was included. The cone angles, correlation times, and diffusion constants obtained through this analysis are shown in Table IV. The inertial and total cone angles, $\theta_{i n}$ and $\theta_{\text {tot }}$, in Table IV were obtained from the inertial order parameter, $T$, and total order parameter, $Q_{t o t}=T S$. The biexponential fit time constants, $t_{1}$ and $t_{2}$ (Table III), are related to the orientational correlation time constants by $t_{1}=\left(1 / \tau_{c}+1 / \tau_{m}\right)^{-1}$ and $t_{2}=\tau_{m}$, which is verified by expanding Eq. (12) into a sum of two exponentials. Corresponding values from the simulated observable are also provided in Table IV.

The orientational relaxation of $\mathrm{SeCN}^{-}$in $\mathrm{D}_{2} \mathrm{O}$ primarily differs from the reorientation of HOD in $\mathrm{D}_{2} \mathrm{O}$ with respect to the wobbling motion, which occurs in a cone of half angle $21.5^{\circ}$ (Table IV). The complete anisotropy decay of HOD decays as a single exponential with a time constant of $3 \pm 0.2$ ps following the ultrafast inertial component. ${ }^{1,2}$ The anisotropy decay in $\mathrm{H}_{2} \mathrm{O}$ has a time constant of $2.6 \pm 0.1$ ps. ${ }^{62}$ In pure water with dilute HOD, orientational relaxation is a concerted process that involves the first and second solvation shells of a particular water molecule. The reorientation occurs through "jump" reorientation, in which multiple hydrogen bonds rearrange and result in a large angular change for a particular hydroxyl of $\sim 60^{\circ} .{ }^{16,63,64}$ The complete orientational randomization of water occurs with a single time constant in contrast to $\mathrm{SeCN}^{-}$, for which the anisotropy decay is biexponential with a wobbling component followed by complete orientational relaxation. The time scale for complete orientational randomization of $\mathrm{SeCN}^{-}$is $4.5 \mathrm{ps}$ (Table IV). $\mathrm{SeCN}^{-}$is a larger molecule than HOD. Simply based on the difference in size, we would expect $\mathrm{SeCN}^{-}$to rotate more slowly. Despite the differences in the functional form of the orientational correlation functions for $\mathrm{HOD}$ and $\mathrm{SeCN}^{-}$, the time scales are not drastically different.

\section{Spectral diffusion}

Figure 7 (left panels) displays the isotropic $(\langle X X X X\rangle$ $+2\langle X X Y Y\rangle) 2 \mathrm{D}$ IR spectra of $\mathrm{SeCN}^{-}$in $\mathrm{D}_{2} \mathrm{O}$ at $T_{w}=0.5$ and 8.8 ps. The main bands in the two panels (red, positive going) arise from the $0 \rightarrow 1$ transition. Below the main bands, a portion of the bands that arise from vibrational echo emission from the $1 \rightarrow 2$ transition (blue, negative going) can be seen. Spectral diffusion of the CN stretch of $\mathrm{SeCN}^{-}$can be extracted by analyzing the timeevolution of the $0-1$ band shape. ${ }^{14}$ At early times (top panel), the band is elongated from the upper right to lower left, reflecting a significant degree of correlation between the initially labeled oscillator frequencies and the final frequencies observed a time $T_{w}$ later. As the waiting time increases (bottom panel), the ensemble of $\mathrm{SeCN}^{-}$anions samples an increasing fraction of $\mathrm{D}_{2} \mathrm{O}$ solvent configurations that are responsible for inhomogeneous broadening of the linear absorption spectrum. The result is an increasingly round 0-1 band shape, reflecting loss of frequency correlation.

The simulated isotropic 2D IR spectra, shown in Fig. 7 (right panels), are in good agreement with the measured results. In particular, the same evolution from an elongated 0-1 band shape at short times ( $\left.T_{w}=0.5 \mathrm{ps}\right)$ to a round band shape at long times $\left(T_{w}=8.8 \mathrm{ps}\right)$ is observed. The minor differences with the experimental spectra are found in the peak position, which is slightly blue shifted in the simulated results, and a slightly greater ellipticity in the band shape.

The time-dependence of the 0-1 band shape can be quantitatively evaluated using the center line slope (CLS) method. ${ }^{65,66}$ The CLS method, applied along the $\omega_{\mathrm{m}}$ axis, involves taking 1D slices of the 0-1 transition parallel to the $\omega_{\mathrm{m}}$ axis over a range of $\omega_{\tau}$ near the band center. Each 1D slice is a spectrum. When the maxima of all 1D slices are plotted, they form a line, the center line, which has a slope, the CLS. As $T_{w}$ is increased, the shape of the $2 \mathrm{D}$ spectrum changes, altering the CLS. The CLS provides detailed information on spectral diffusion. In principle, the CLS begins at an initial value of 1 (perfect correlation) and decays to a value of zero when spectral diffusion is complete (perfectly uncorrelated). In practice, the CLS begins at some value less
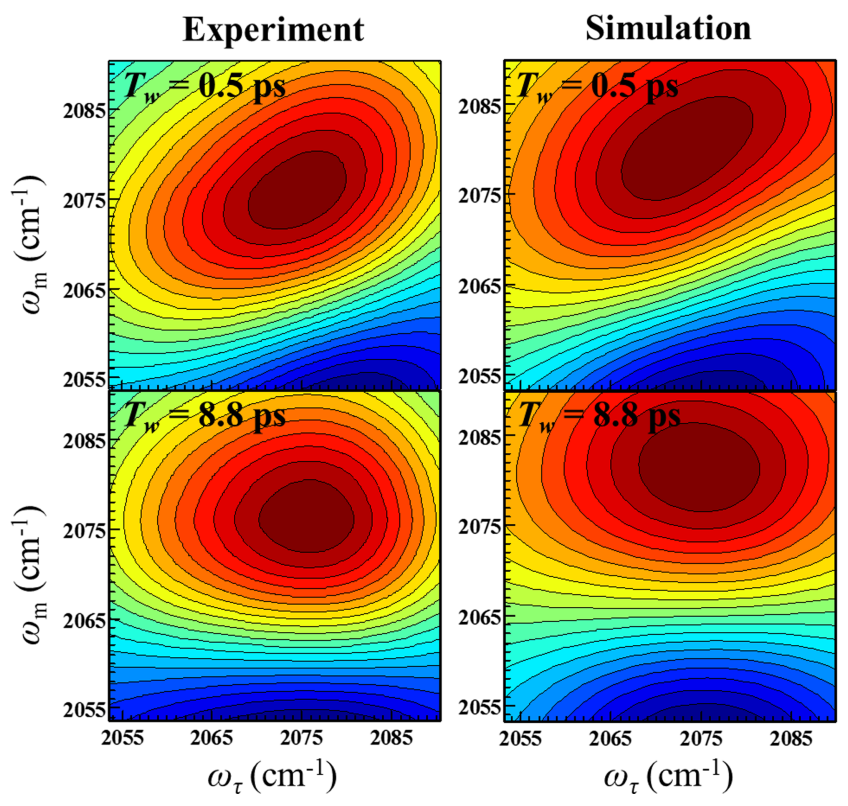

FIG. 7. Representative experimental (left) and simulated (right) isotropic 2D IR spectra of the $\mathrm{CN}$ stretch of $\mathrm{SeCN}^{-}$in $\mathrm{D}_{2} \mathrm{O}$ at $T_{w}=0.5 \mathrm{ps}$ and $8.8 \mathrm{ps}$. The 2D spectrum changes shape as spectral diffusion proceeds with increasing $T_{w}$. 
than 1 due to the presence of the homogeneous linewidth, which is $T_{w}$ independent, as a source of spectral broadening. ${ }^{65}$ Greater homogeneous broadening manifests as a larger difference between the initial value of the CLS and 1. These features are evident in the $\langle X X X X\rangle$ (black points), $\langle X X Y Y\rangle$ (red points), and $\langle X X X X\rangle+2\langle X X Y Y\rangle$ (blue points) CLS curves obtained from the data and shown in Fig. 8(a). They can also be observed in the simulated CLS curve (red points), which is compared to the measured isotropic result (blue points) in Fig. 8(b).

The CLS is equivalent to the normalized frequencyfrequency correlation function. ${ }^{65,66}$ The FFCF is the probability that an oscillator with an initial frequency will have the same frequency a time $t$ later, averaged over all initial frequencies in the inhomogeneous spectral distribution. Here the FFCF was modeled with a simplified Kubo form, ${ }^{67}$

$$
C(t)=\langle\delta \omega(t) \delta \omega(0)\rangle=\sum_{i} \Delta_{i}^{2} \exp \left[-t / \tau_{i}\right],
$$

where $\delta \omega(t)=\omega(t)-\langle\omega\rangle$ is the fluctuation of the instantaneous frequency, $\omega(t)$, from the average frequency, $\langle\omega\rangle . \Delta_{i}$

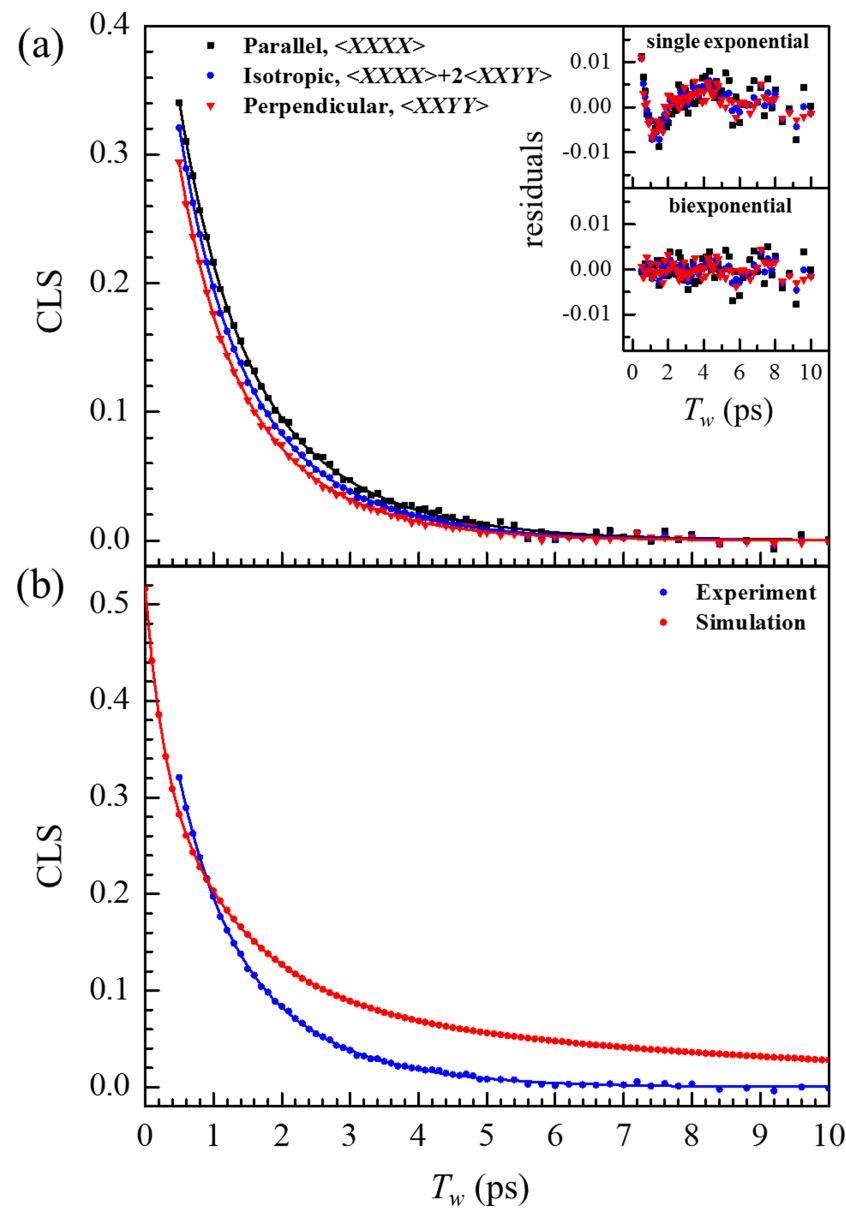

FIG. 8. (a) Parallel, isotropic, and perpendicular CLS decays (spectral diffusion) for the $\mathrm{CN}$ stretch of $\mathrm{SeCN}^{-}$in $\mathrm{D}_{2} \mathrm{O}$. The solid curves are biexponential fits to the data. The top and bottom insets display the residuals obtained with single and biexponential fits to the data, respectively. (b) Simulated CLS decay (red circles) compared to the measured CLS decay (blue circles) for the CN stretch of $\mathrm{SeCN}^{-}$in $\mathrm{D}_{2} \mathrm{O}$. The fits described in the text are shown as solid curves of the same color. and $\tau_{i}$ are the frequency fluctuation amplitude and time constant, respectively, of the $i$ th component. A component of the FFCF is motionally narrowed and a source of homogeneous broadening in the absorption line if $\Delta \times \tau \ll 1 .^{11}$ When a component is motionally narrowed, it is not possible to determine $\Delta$ and $\tau$ separately. The motionally narrowed contribution to the absorption spectrum has a pure dephasing linewidth given by $\Gamma^{*}=\Delta^{2} \tau / \pi=1 / \pi T_{2}^{*}$, where $T_{2}^{*}$ is the pure dephasing time. The observed total homogeneous dephasing time is

$$
\frac{1}{T_{2}}=\frac{1}{T_{2}^{*}}+\frac{1}{2 T_{1}}+\frac{1}{3 T_{o r}},
$$

where $T_{1}$ is the vibrational lifetime and $T_{\text {or }}$ is the orientational relaxation time. ${ }^{65}$ Therefore, the homogeneous (Lorentzian) linewidth is $\Gamma=1 / \pi T_{2}$. The homogeneous broadening is reflected in the difference from 1 of the CLS value at $T_{w}$ $=0$ (see Fig. 8). By combining the CLS decay data with the absorption spectrum, the homogeneous component and the full FFCF are obtained. ${ }^{65,66}$ The lifetime, $T_{1}\left(0.1 \mathrm{~cm}^{-1}\right)$, and orientational relaxation, $T_{\text {or }}\left(0.8 \mathrm{~cm}^{-1}\right)$, combined contribute less than $1 \mathrm{~cm}^{-1}$ to the total homogeneous linewidth, which is $\sim 8 \mathrm{~cm}^{-1}$ (see Table VI). Therefore, the homogeneous linewidth is dominated by motionally narrowed pure dephasing. Each $\Delta_{i}$ in Eq. (13) is the standard deviation of the Gaussian line shape associated with the $i$ th component of the inhomogeneous (Gaussian) line shape. The inhomogeneous component of the absorption spectrum is the convolution of the Gaussian components so that its standard deviation for two inhomogeneous components (see below) is $\Delta_{\text {total }}=\left(\Delta_{1}^{2}+\Delta_{2}^{2}\right)^{1 / 2}$, and the FWHM is $2[2 \ln (2)]^{1 / 2} \Delta_{\text {total }}$ $\left(2.35 \Delta_{\text {total }}\right)$. The linear absorption line shape (Voigt profile) is the convolution of the homogeneous and inhomogeneous line shapes. The CLS therefore establishes the relative contribution of homogeneous broadening and each component of the inhomogeneous broadening to the total line shape. By simultaneously fitting the CLS and linear absorption spectrum, the FFCF can be determined in absolute frequency units $(\Delta \mathrm{s}$ in $\left.\mathrm{cm}^{-1}\right) .65$

The CLS points shown in Fig. 8(a) are the mean of the experimental results for 6 independently prepared samples. The residuals, corresponding to single and biexponential fits to the data, are shown in the insets of Fig. 8(a). As with the anisotropy curves, the CLS decays are clearly biexponential. The CLS decays are slightly different for the three polarization configurations [Fig. 8(a)]. This is an indication that the frequency fluctuations and rotational motion of the vibrational probe are coupled to a small extent. ${ }^{68-70}$ The contributions to spectral diffusion from the structural evolution of the sample (structural spectral diffusion, SSD) and from reorientation induced spectral diffusion (RISD) have been discussed in detail, and under appropriate conditions they can be separated. ${ }^{69,70}$ However, the differences in the spectral diffusion parameters given in Tables V and VI for the three polarization configurations are all within the error bars of each other. Therefore, the RISD contribution is very small and will not be considered. We take the data and the values in Tables $\mathrm{V}$ and VI to describe the SSD. The MD simulations, which do not include the polarized radiation fields, calculate the isotropic data and include all contributions to spectral diffusion. 
TABLE V. CLS fit parameters. $A_{i}$ and $\tau_{i}$ are the amplitude and time constant of the $i$ th component of the multi-exponential fit to the CLS data, respectively.

\begin{tabular}{lcccccc}
\hline \hline & $A_{1}$ & $\tau_{1}(\mathrm{ps})$ & $A_{2}$ & $\tau_{2}(\mathrm{ps})$ & $A_{3}$ & $\tau_{3}(\mathrm{ps})$ \\
\hline Parallel & $0.27 \pm 0.03$ & $0.66 \pm 0.09$ & $0.30 \pm 0.04$ & $1.6 \pm 0.1$ & & \\
Isotropic & $0.25 \pm 0.03$ & $0.6 \pm 0.1$ & $0.31 \pm 0.04$ & $1.4 \pm 0.2$ & & \\
Perpendicular & $0.22 \pm 0.02$ & $0.5 \pm 0.1$ & $0.30 \pm 0.04$ & $1.3 \pm 0.2$ & & \\
Simulation & $0.27 \pm 0.01$ & 0.6 & $0.11 \pm 0.01$ & 1.4 & $0.11 \pm 0.01$ & $6.9 \pm 0.2$ \\
\hline \hline
\end{tabular}

The isotropic CLS shows fast $0.6 \pm 0.1$ ps and slow 1.4 \pm 0.2 ps components that are observed with nearly equal relative amplitudes (Table V). The spectral diffusion of $\mathrm{SeCN}^{-}$was previously reported to be a single 1.5 ps exponential decay. ${ }^{49}$ Improvements in the signal to noise ratio and time resolution achieved in these experiments permitted the observation of this additional fast time scale in the spectral diffusion. The absolute FFCFs, with time scales in ps and homogeneous linewidths and frequency fluctuation amplitudes in $\mathrm{cm}^{-1}$, are given in Table VI. The hydrogen bond dynamics observed using dilute HOD as the vibrational probe in water occur on two time scales, $\sim 0.34$ ps and $1.4 \pm 0.2 \mathrm{ps}$ in $\mathrm{D}_{2} \mathrm{O}^{1}$ and $0.4 \pm 0.1 \mathrm{ps}$ and $1.7 \pm 0.2 \mathrm{ps}$ in $\mathrm{H}_{2} \mathrm{O} \cdot{ }^{6,12,13}$ It is remarkable that the spectral diffusion of the $\mathrm{CN}$ stretch of the anion, $\mathrm{SeCN}^{-}$, is virtually identical to the spectral diffusion observed with isotopically substituted water (HOD) in water. In pure water, MD simulations have identified the fast, $\sim 0.4 \mathrm{ps}$ component, as arising from very local hydrogen bond length and, to some extent, angle fluctuations, and the slow component is caused by the complete randomization of the hydrogen bond network. ${ }^{1,12}$ These results indicate that the spectral diffusion sensed by $\mathrm{SeCN}^{-}$is caused by water hydrogen bond dynamics and that the presence of the anion does not significantly change the dynamics of water in the proximity of the anion from those of pure water. The $\mathrm{OH}$ of HOD is a hydrogen bond donor, while the $\mathrm{CN}$ of $\mathrm{SeCN}^{-}$is a hydrogen bond acceptor. Despite this difference, they both are effectively local modes with frequency fluctuations determined by the dynamics of the surrounding water.

The detailed nature of the dynamics that gives rise to the spectral diffusion sensed by $\mathrm{SeCN}^{-}$is explicated by the MD simulations. The FFCF can be calculated directly from the simulations. From the simulated FFCF, a series of absorptive 2D spectra were calculated as a function of $T_{w}$ with the $3 \mathrm{rd}$ order response functions, summing contributions from nonrephasing and rephasing pathways, and making the Condon and second order cumulant expansion truncation approximations. ${ }^{8}$ These spectra were treated as data and the CLS was calculated in the same manner as used for the experimental 2D IR spectra. The simulated CLS [Fig. 8(b), red points] is fit well by a multi-exponential function. The two experimentally observed decay time constants are reproduced essentially perfectly, with the simulated spectral diffusion accurately described by the time constants of 0.6 and $1.4 \mathrm{ps}$ (see Table V). However, in addition to the simulation results that reproduce the experimental data, the simulations yield a small slow decay component with a time constant of 6.9 ps and an amplitude of 0.1 . This long-time decay is not found in the measurements and appears to be a minor defect of the model. However, the simulations provide a very reasonable quantitative description of the measured spectral diffusion time scales.

In addition, the simulation yields a large homogeneous component with $\tau=90$ fs and $\Delta=19 \mathrm{~cm}^{-1} . \Delta$ and $\tau$ of the ultrafast motionally narrowed component cannot be measured separately [see Eq. (14) and the discussion below Eq. (13)]. From the experiments, the homogeneous linewidth $\Gamma=1 / \pi T_{2}$ is determined. $T_{2}$ is dominated by the pure dephasing, $T_{2}^{*}$. The comparison between the simulated and experimental $\Gamma$ 's and the remaining FFCF parameters are contained in Table VI.

It is worth noting that, because the map is linear in the field, it is readily demonstrated that the long time scale decay observed in the simulated CLS does not depend on the map parameters. That is, if the map is linear in the field, $\omega_{01}$ $=c_{0}+c_{1} \varepsilon_{c}$, then it is straightforward to show that the normalized FFCF can be written as $\left\langle\delta \varepsilon_{c}(0) \delta \varepsilon_{c}(t)\right\rangle /\left\langle\delta \varepsilon_{c}(0) \delta \varepsilon_{c}(0)\right\rangle$ and is thus independent of the map parameters, $c_{0}$ and $c_{1}$. This consideration suggests that the FFCF reports on the structural rearrangements of the waters solvating $\mathrm{SeCN}^{-}$, which are determined by the force field. However, the possibility that the FFCF may exhibit effects due to the inaccuracy of the linear empirical map description is not fully eliminated.

We can examine the origins of the spectral diffusion time scales by further analysis of the simulated FFCF and the

TABLE VI. FFCF parameters. $T_{2}$ and $\Gamma$ are the total homogeneous dephasing time and homogeneous linewidth (FWHM), respectively. $\Delta_{i}$ and $\tau_{i}$ are the absolute frequency fluctuation amplitude and time constant of the $i$ th component of the inhomogeneous line shape, respectively.

\begin{tabular}{lcrrccccc}
\hline \hline & $T_{2}(\mathrm{ps})$ & $\Gamma\left(\mathrm{cm}^{-1}\right)$ & $\Delta_{1}\left(\mathrm{~cm}^{-1}\right)$ & $\tau_{1}(\mathrm{ps})$ & $\Delta_{2}\left(\mathrm{~cm}^{-1}\right)$ & $\tau_{2}(\mathrm{ps})$ & $\Delta_{3}\left(\mathrm{~cm}^{-1}\right)$ & $\tau_{3}(\mathrm{ps})$ \\
\hline Parallel & $1.4 \pm 0.1$ & $7.6 \pm 0.7$ & $9.7 \pm 0.7$ & $0.66 \pm 0.09$ & $10.0 \pm 0.5$ & $1.6 \pm 0.1$ & & \\
Isotropic & $1.4 \pm 0.1$ & $7.6 \pm 0.2$ & $9.7 \pm 0.5$ & $0.6 \pm 0.1$ & $10.2 \pm 0.8$ & $1.4 \pm 0.2$ & & \\
Perpendicular & $1.2 \pm 0.1$ & $8.7 \pm 0.4$ & $9.4 \pm 0.5$ & $0.5 \pm 0.1$ & $10.2 \pm 0.9$ & $1.3 \pm 0.2$ & & \\
Simulation & $0.8 \pm 0.1$ & $13.0 \pm 0.1$ & $13.2 \pm 0.1$ & 0.6 & $6.0 \pm 0.1$ & 1.4 & $5.6 \pm 0.1$ & $7.1 \pm 0.1$ \\
\hline \hline
\end{tabular}


hydrogen bond dynamics around the $\mathrm{SeCN}^{-}$solute. Figure 4 shows that there is a strong relationship between the $\mathrm{CN}$ stretching frequency and the number of hydrogen bonds to $\mathrm{SeCN}^{-}$. It is then interesting to consider how the time scales for spectral diffusion compare with those for hydrogen bond dynamics. The latter can be examined by the survival probability, $P_{H B}(t)$, for OD currently hydrogen bonded to $\mathrm{SeCN}^{-}$ to still be hydrogen bonded to the solute at some later time. The survival probability calculated as just described includes OD groups that break a hydrogen bond to $\mathrm{SeCN}^{-}$but reform it at a later time. An alternative approach is to focus on the first hydrogen-bond breaking event by setting the contribution to the survival probability for a given hydrogen bond donor to zero after it is broken for a time interval $\tau_{a b s}$, where the subscript notes that this is a type of absorbing boundary condition. This survival probability, which we denote as $P_{H B}\left(t ; \tau_{a b s}\right)$, recovers $P_{H B}(t)$ as $\tau_{a b s} \rightarrow \infty$, and as $\tau_{a b s} \rightarrow 0$ gives the survival probability for a continuous hydrogen bond. In addition to calculating $P_{H B}(t)$, we have taken $\tau_{a b s}=0.2 \mathrm{ps}$, which prevents contributions to the decay of the survival probability from transient hydrogen bond breaks (i.e., events where the OD group breaks the hydrogen bond fleetingly before returning to the original acceptor), which are known to occur on the time scale of $0.2-0.25$ ps in water. ${ }^{63,71}$

The two approaches for calculating hydrogen bond survival probabilities are shown in Fig. 9. The result for $P_{H B}\left(t ; \tau_{a b s}=0.2 \mathrm{ps}\right)$ (red curve) is described by a small, initial rapid decay on a $0.28 \mathrm{ps}$ time scale followed by a $3.35 \mathrm{ps}$ decay. This is consistent with the time scale of 3.1-3.3 ps for hydrogen bond exchanges found in neat water, ${ }^{64,71}$ suggesting that $\mathrm{OD}$ groups hydrogen bonded to $\mathrm{SeCN}^{-}$have very similar dynamics as those hydrogen bonded to other waters, making the anion an excellent reporter. Note that, as with neat water, the spectral diffusion takes place on a faster time scale than that for hydrogen bond exchanges.

The survival probability without absorbing boundary conditions, $P_{H B}(t)$ (blue curve), exhibits an additional, longer time

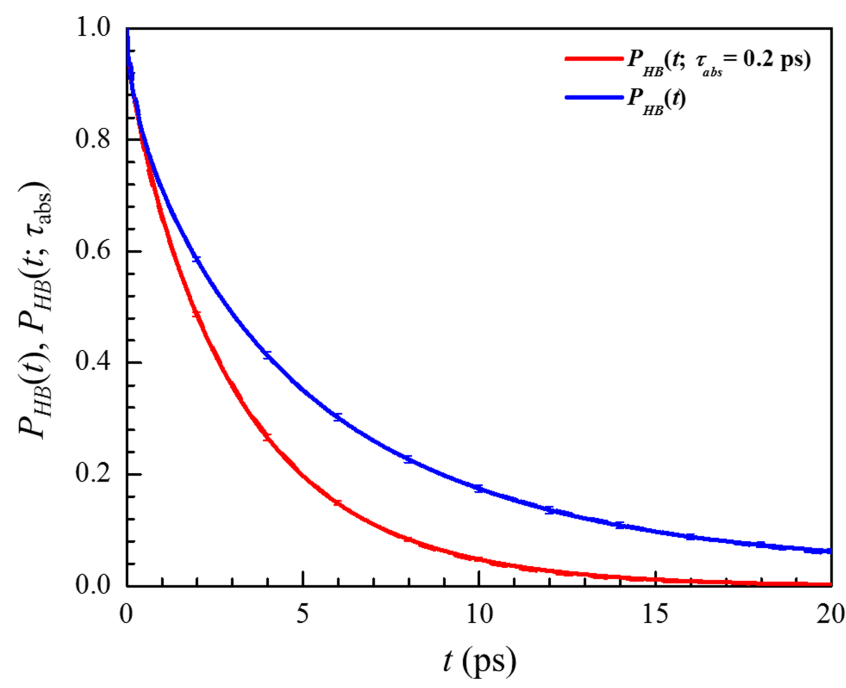

FIG. 9. Survival probabilities with (red curve) and without (blue curve) absorbing boundary conditions for hydrogen bonds between OD groups and $\mathrm{SeCN}^{-}$as a function of time. The fits described in the text are shown as dashed curves of the same color, but are indistinguishable from the data. scale, as is evident in Fig. 9. The decay can be fit using the same two time scales obtained by fitting $P_{H B}\left(t ; \tau_{a b s}=0.2 \mathrm{ps}\right)$ and a third component with a decay time of 10.2 ps. Because the only difference in the two survival probabilities involves the reformation of hydrogen bonds that are broken, this longest time scale can be attributed to waters leaving the first solvation shell of the $\mathrm{SeCN}^{-}$anion. While an analogous longer time scale appears in the simulated CLS, no such component is present in the measured CLS. The lack of a long time scale component in the experimental CLS suggests that the model overestimates the barrier for waters to leave the $\mathrm{SeCN}^{-}$solvation shell, indicating a direction for improving upon the present approach. In particular, it will be important to examine whether including polarizability or charge transfer effects change this aspect of the dynamics. The absence of the slow component in the current experiments that is present in the simulations further implies that the experiments are consistent with a picture where solvating waters leave the first solvation shell of $\mathrm{SeCN}^{-}$following a single hydrogen bond exchange.

\section{CONCLUDING REMARKS}

The sensitivity of the $\mathrm{SeCN}^{-}$vibrational probe to water hydrogen bond dynamics has been analyzed with ultrafast IR experiments and simulations. Selenocyanate has an asymmetric absorption line shape with broadening on the lower frequency side of the line [Fig. 2(a), black curve]. The observation of spectral diffusion in the isotropic pump-probe decays (Fig. 5, inset) suggests that the line broadening is the result of a frequency-dependent transition dipole moment, i.e., the non-Condon effect. The formation of a strong axial hydrogen bond to the nitrogen blue shifts the absorption relative to no hydrogen bonding, but additional weak hydrogen bonds result in a red shift in the resonance frequency, an increase in the transition dipole strength, ${ }^{49}$ and perhaps reduced motional narrowing. This leads to excess absorption on the red side of the band in the pump-probe experiment. The simulated spectrum [Fig. 2(b), blue curve] does not exhibit the asymmetric line shape, but does show stronger transition dipole moments for red-shifted frequencies due to more, weaker hydrogen bonding interactions with solvating waters. The net flow of population from red to blue frequencies, seen in the early time pump-probe data, is caused by spectral diffusion as oscillators sample different frequencies within the inhomogeneous line shape.

The PSPP experiments and simulations (Fig. 6) both show that the complete orientational randomization of $\mathrm{SeCN}^{-}$ in $\mathrm{D}_{2} \mathrm{O}$ has a 4.5 ps time constant (Table IV). In contrast to $\mathrm{HOD}, \mathrm{SeCN}^{-}$also exhibits fast wobbling-in-a-cone restricted angular sampling ( $2.0 \mathrm{ps})$ with a cone half-angle of $21.5^{\circ}$ (Table IV). Following the ultrafast inertial orientational motions, the observation of wobbling-in-a-cone indicates that certain intermolecular interactions between the anion and the hydrogen bond network restrict the reorientation of $\mathrm{SeCN}^{-}$, while no such barriers appear to limit rotation of HOD. However, the structural restrictions relax very quickly as the hydrogen bond network proceeds to randomize about the anion. 
Finally, the results presented here demonstrate that $\mathrm{SeCN}^{-}$ is a reliable probe of hydrogen bond rearrangement dynamics in water. Although HOD and $\mathrm{SeCN}^{-}$differ with respect to their charge and hydrogen bond donating or accepting roles in $\mathrm{D}_{2} \mathrm{O}$, their FFCFs are remarkably similar. The $0.6 \pm 0.1$ and $1.4 \pm 0.2$ ps components of the $\mathrm{SeCN}^{-}$spectral diffusion (Tables V and VI), like the $\sim 0.34$ and $1.4 \pm 0.2$ ps components in the spectral diffusion of HOD, ${ }^{1}$ correspond to local fluctuations in hydrogen bond geometries and large-scale rearrangement of the extended hydrogen bond network surrounding the probe, respectively.

The observation of virtually the same exponential decays in the FFCFs of both vibrational probes in $\mathrm{D}_{2} \mathrm{O}$ suggests a promising pathway for future investigations of water structure and dynamics in confined systems, in which the dynamics are predicted to be dramatically slower than in bulk water. The impact of confinement on the dynamics of solvents and solutes is an important problem in the field of heterogeneous catalysis, ${ }^{24}$ and many other problems in chemistry, ${ }^{72}$ biology, ${ }^{73}$ and materials science. ${ }^{74}$ The strong transition dipole and significantly longer lifetime of $\mathrm{SeCN}^{-}$compared to HOD means that it can serve as a sensitive probe of water dynamics in future investigations of complex heterogeneous materials.

\section{ACKNOWLEDGMENTS}

This work was funded by the Division of Chemical Sciences, Geosciences, and Biosciences, Office of Basic Energy Sciences of the U.S. Department of Energy through Grant Nos. DE-FG03-84ER13251 (S.A.Y. and M.D.F.) and DEFG02-05ER15708 (W.H.T.). Additional support of the 2D IR instrument and for M.D.F. was provided by Air Force Office of Scientific Research Grant No. FA9550-16-1-0104. The simulations were carried out at the University of Kansas Advanced Computing Facility.

${ }^{1}$ C. J. Fecko, J. J. Loparo, S. T. Roberts, and A. Tokmakoff, "Local hydrogen bonding dynamics and collective reorganization in water: Ultrafast infrared spectroscopy of HOD/D ${ }_{2} \mathrm{O}$," J. Chem. Phys. 122, 054506 (2005).

${ }^{2}$ J. J. Loparo, C. J. Fecko, J. D. Eaves, S. T. Roberts, and A. Tokmakoff, "Reorientational and configurational fluctuations in water observed on molecular length scales,” Phys. Rev. B 70, 180201 (2004).

${ }^{3}$ Y. L. A. Rezus and H. J. Bakker, "On the orientational relaxation of HDO in liquid water," J. Chem. Phys. 123, 114502 (2005).

${ }^{4}$ A. Shimizu and Y. Taniguchi, "NMR studies on reorientational motion of hydrated $\mathrm{D}_{2} \mathrm{O}$ molecules of halide ions $\left(\mathrm{F}^{-}, \mathrm{Cl}^{-}, \mathrm{Br}^{-}\right.$, and $\left.\mathrm{I}^{-}\right)$in dilute aqueous solutions,” Bull. Chem. Soc. Jpn. 64, 1613-1617 (1991).

${ }^{5}$ C. Rønne, P.-O. Åstrand, and S. R. Keiding, "THz spectroscopy of liquid $\mathrm{H}_{2} \mathrm{O}$ and $\mathrm{D}_{2} \mathrm{O}$," Phys. Rev. Lett. 82, 2888-2891 (1999).

${ }^{6}$ J. B. Asbury, T. Steinel, K. Kwak, S. A. Corcelli, C. P. Lawrence, J. L. Skinner, and M. D. Fayer, "Dynamics of water probed with vibrational echo correlation spectroscopy," J. Chem. Phys. 121, 12431-12446 (2004).

${ }^{7}$ S. A. Corcelli, C. P. Lawrence, and J. L. Skinner, "Combined electronic structure/molecular dynamics approach for ultrafast infrared spectroscopy of dilute $\mathrm{HOD}$ in liquid $\mathrm{H}_{2} \mathrm{O}$ and $\mathrm{D}_{2} \mathrm{O}$," J. Chem. Phys. 120, 8107-8117 (2004).

${ }^{8}$ J. R. Schmidt, S. A. Corcelli, and J. L. Skinner, "Pronounced non-Condon effects in the ultrafast infrared spectroscopy of water," J. Chem. Phys. 123, 044513 (2005).

${ }^{9}$ M. Koziński, S. Garrett-Roe, and P. Hamm, "Vibrational spectral diffusion of $\mathrm{CN}^{-}$in water," Chem. Phys. 341, 5-10 (2007).

${ }^{10}$ M. W. Lee, J. K. Carr, M. Göllner, P. Hamm, and M. Meuwly, "2D IR spectra of cyanide in water investigated by molecular dynamics simulations," J. Chem. Phys. 139, 054506 (2013).
${ }^{11} \mathrm{P}$. Hamm and M. T. Zanni, Concepts and Methods of $2 D$ Infrared Spectroscopy (Cambridge University Press, New York, 2011).

${ }^{12}$ J. B. Asbury, T. Steinel, C. Stromberg, S. A. Corcelli, C. P. Lawrence, J. L. Skinner, and M. D. Fayer, "Water dynamics: Vibrational echo correlation spectroscopy and comparison to molecular dynamics simulations," J. Phys. Chem. A 108, 1107-1119 (2004).

${ }^{13}$ S. Park and M. D. Fayer, "Hydrogen bond dynamics in aqueous $\mathrm{NaBr}$ solutions," Proc. Natl. Acad. Sci. U. S. A. 104, 16731-16738 (2007).

${ }^{14}$ S. Park, K. Kwak, and M. D. Fayer, "Ultrafast 2D-IR vibrational echo spectroscopy: A probe of molecular dynamics," Laser Phys. Lett. 4, 704 (2007).

${ }^{15}$ C. J. Fecko, J. D. Eaves, J. J. Loparo, A. Tokmakoff, and P. L. Geissler, "Ultrafast hydrogen-bond dynamics in the infrared spectroscopy of water," Science 301, 1698-1702 (2003).

${ }^{16} \mathrm{D}$. Laage and J. T. Hynes, "Reorientional dynamics of water molecules in anionic hydration shells," Proc. Natl. Acad. Sci. U. S. A. 104, 11167-11172 (2007).

${ }^{17}$ Y.-S. Lin, B. M. Auer, and J. L. Skinner, "Water structure, dynamics, and vibrational spectroscopy in sodium bromide solutions," J. Chem. Phys. 131, 144511 (2009).

${ }^{18}$ C. H. Giammanco, D. B. Wong, and M. D. Fayer, "Water dynamics in divalent and monovalent concentrated salt solutions," J. Phys. Chem. B 116, 13781-13792 (2012).

${ }^{19}$ H. Bian, J. Li, Q. Zhang, H. Chen, W. Zhuang, Y. Q. Gao, and J. Zheng, "Ion segregation in aqueous solutions," J. Phys. Chem. B 116, 14426-14432 (2012).

${ }^{20}$ H. Bian, H. Chen, Q. Zhang, J. Li, X. Wen, W. Zhuang, and J. Zheng, "Cation effects on rotational dynamics of anions and water molecules in alkali $\left(\mathrm{Li}^{+}\right.$, $\mathrm{Na}^{+}, \mathrm{K}^{+}, \mathrm{Cs}^{+}$) thiocyanate $\left(\mathrm{SCN}^{-}\right)$aqueous solutions," J. Phys. Chem. B 117, 7972-7984 (2013).

${ }^{21}$ G. Stirnemann, E. Wernersson, P. Jungwirth, and D. Laage, "Mechanisms of acceleration and retardation of water dynamics by ions," J. Am. Chem. Soc. 135, 11824-11831 (2013).

${ }^{22}$ C. H. Giammanco, P. L. Kramer, and M. D. Fayer, "Dynamics of dihydrogen bonding in aqueous solutions of sodium borohydride," J. Phys. Chem. B 119, 3546-3559 (2015).

${ }^{23}$ P. C. Burris, D. Laage, and W. H. Thompson, "Simulations of the infrared, Raman, and 2D-IR photon echo spectra of water in nanoscale silica pores," J. Chem. Phys. 144, 194709 (2016).

${ }^{24} \mathrm{D}$. Laage and W. H. Thompson, "Reorientation dynamics of nanoconfined water: Power-law decay, hydrogen-bond jumps, and test of a two-state model," J. Chem. Phys. 136, 044513 (2012).

${ }^{25} \mathrm{~S}$. Woutersen and H. J. Bakker, "Resonant intermolecular transfer of vibrational energy in liquid water," Nature 402, 507-509 (1999).

${ }^{26}$ K. J. Gaffney, I. R. Piletic, and M. D. Fayer, "Orientational relaxation and vibrational excitation transfer in methanol-carbon tetrachloride solutions," J. Chem. Phys. 118, 2270-2278 (2003).

${ }^{27}$ S. K. Karthick Kumar, A. Tamimi, and M. D. Fayer, "Comparisons of 2D IR measured spectral diffusion in rotating frames using pulse shaping and in the stationary frame using the standard method," J. Chem. Phys. 137, 184201 (2012).

${ }^{28} \mathrm{~S} .-\mathrm{H}$. Shim and M. T. Zanni, "How to turn your pump-probe instrument into a multidimensional spectrometer: 2D IR and Vis spectroscopies via pulse shaping," Phys. Chem. Chem. Phys. 11, 748-761 (2009).

${ }^{29}$ S.-H. Shim, D. B. Strasfeld, E. C. Fulmer, and M. T. Zanni, "Femtosecond pulse shaping directly in the mid-IR using acousto-optic modulation," Opt. Lett. 31, 838-840 (2006).

${ }^{30} \mathrm{~A}$. Tokmakoff, "Orientational correlation functions and polarization selectivity for nonlinear spectroscopy of isotropic media. I. Third order," J. Chem. Phys. 105, 1-12 (1996).

${ }^{31}$ H.-S. Tan, I. R. Piletic, and M. D. Fayer, "Polarization selective spectroscopy experiments: Methodology and pitfalls,” J. Opt. Soc. Am. B 22, 2009-2017 (2005).

${ }^{32}$ J. Nishida, A. Tamimi, H. Fei, S. Pullen, S. Ott, S. M. Cohen, and M. D. Fayer, "Structural dynamics inside a functionalized metal-organic framework probed by ultrafast 2D IR spectroscopy," Proc. Natl. Acad. Sci. U. S. A. 111, 18442-18447 (2014).

${ }^{33}$ H. J. C. Berendsen, J. R. Grigera, and T. P. Straatsma, "The missing term in effective pair potentials," J. Phys. Chem. 91, 6269-6271 (1987).

${ }^{34}$ A. V. Marenich, C. J. Cramer, and D. G. Truhlar, "Universal solvation model based on solute electron density and on a continuum model of the solvent defined by the bulk dielectric constant and atomic surface tensions," J. Phys. Chem. B 113, 6378-6396 (2009). 
${ }^{35}$ P. J. Gee and W. F. van Gunsteren, "Acetonitrile revisited: A molecular dynamics study of the liquid phase," Mol. Phys. 104, 477-483 (2006).

${ }^{36}$ C. J. Fennell and J. D. Gezelter, "Is the Ewald summation still necessary? Pairwise alternatives to the accepted standard for long-range electrostatics," J. Chem. Phys. 124, 234104 (2006).

${ }^{37}$ J.-P. Ryckaert, G. Ciccotti, and H. J.C. Berendsen, "Numerical integration of the cartesian equations of motion of a system with constraints: Molecular dynamics of $n$-alkanes," J. Comput. Phys. 23, 327-341 (1977).

${ }^{38} \mathrm{~S}$. Nose, "A molecular dynamics method for simulations in the canonical ensemble," Mol. Phys. 52, 255-268 (1984).

${ }^{39}$ W. G. Hoover, "Canonical dynamics: Equilibrium phase-space distributions," Phys. Rev. A 31, 1695-1697 (1985).

${ }^{40}$ D. P. Shoemaker, C. W. Garland, and J.W. Nibler, Experiments in Physical Chemistry (McGraw-Hill, New York, 1989).

${ }^{41}$ B. A. Lindquist and S. A. Corcelli, "Nitrile groups as vibrational probes: Calculations of the $\mathrm{C} \equiv \mathrm{N}$ infrared absorption line shape of acetonitrile in water and tetrahydrofuran," J. Phys. Chem. B 112, 6301-6303 (2008).

${ }^{42}$ B. Blasiak, A. W. Ritchie, L. J. Webb, and M. Cho, "Vibrational solvatochromism of nitrile infrared probes: Beyond the vibrational Stark dipole approach," Phys. Chem. Chem. Phys. 18, 18094-18111 (2016).

${ }^{43}$ C. M. Morales and W. H. Thompson, "Simulations of infrared spectra of nanoconfined liquids: Acetonitrile confined in nanoscale, hydrophilic silica pores," J. Phys. Chem. A 113, 1922-1933 (2009).

${ }^{44}$ J.-H. Choi, K.-I. Oh, H. Lee, C. Lee, and M. Cho, "Nitrile and thiocyanate IR probes: Quantum chemistry calculation studies and multivariate least-square fitting analysis," J. Chem. Phys. 128, 134506 (2008).

${ }^{45}$ H. Lee, J.-H. Choi, and M. Cho, "Vibrational solvatochromism and electrochromism of cyanide, thiocyanate, and azide anions in water," Phys. Chem. Chem. Phys. 12, 12658 (2010).

${ }^{46}$ D. T. Colbert and W. H. Miller, "A novel discrete variable representation for quantum mechanical reactive scattering via the $S$-matrix Kohn method," J. Chem. Phys. 96, 1982-1991 (1992).

${ }^{47}$ J. R. Schmidt, S. T. Roberts, J. J. Loparo, A. Tokmakoff, M. D. Fayer, and J. L. Skinner, "Are water simulation models consistent with steadystate and ultrafast vibrational spectroscopy experiments?," Chem. Phys. 341, 143-157 (2007)

${ }^{48}$ P. L. Kramer, C. H. Giammanco, and M. D. Fayer, "Dynamics of water, methanol, and ethanol in a room temperature ionic liquid," J. Chem. Phys. 142, 212408 (2015).

${ }^{49}$ R. Yuan, C. Yan, A. Tamimi, and M. D. Fayer, "Molecular anion hydrogen bonding dynamics in aqueous solution," J. Phys. Chem. B 119, 1340713415 (2015)

${ }^{50}$ P. W. Schultz, G. E. Leroi, and A. I. Popov, "Solvation of $\mathrm{SCN}^{-}$and $\mathrm{SeCN}^{-}$ anions in hydrogen-bonding solvents," J. Am. Chem. Soc. 118, 1061710625 (1996).

${ }^{51}$ B. A. Lindquist, R. T. Haws, and S. A. Corcelli, "Optimized quantum mechanics/molecular mechanics strategies for nitrile vibrational probes: Acetonitrile and para-Tolunitrile in water and tetrahydrofuran," J. Phys. Chem. B 112, 13991-14001 (2008).

${ }^{52} \mathrm{~S}$. A. Corcelli and J. L. Skinner, "Infrared and Raman line shapes of dilute HOD in liquid $\mathrm{H}_{2} \mathrm{O}$ and $\mathrm{D}_{2} \mathrm{O}$ from 10 to $90^{\circ} \mathrm{C}$," J. Phys. Chem. A 109, 6154-6165 (2005)

${ }^{53}$ A. Tamimi and M. D. Fayer, "Ionic liquid dynamics measured with 2D IR and IR pump-probe experiments on a linear anion and the influence of potassium cations," J. Phys. Chem. B 120, 5842-5854 (2016).

${ }^{54}$ J. R. Reimers and L. E. Hall, "The solvation of acetonitrile," J. Am. Chem. Soc. 121, 3730-3744 (1999).
${ }^{55} \mathrm{~T}$. Tao, "Time-dependent fluorescence depolarization and Brownian rotational diffusion coefficients of macromolecules," Biopolymers 8, 609-632 (1969).

${ }^{56}$ T. Steinel, J. B. Asbury, J. Zheng, and M. D. Fayer, "Watching hydrogen bonds break: A transient absorption study of water," J. Phys. Chem. A 108, 10957-10964 (2004).

${ }^{57}$ H. Akaike, "A new look at the statistical model identification," IEEE Trans. Autom. Control 19, 716-723 (1974).

${ }^{58}$ K. Kinosita, S. Kawato, and A. Ikegami, "A theory of fluorescence polarization decay in membranes," Biophys. J. 20, 289-305 (1977).

${ }^{59}$ G. Lipari and A. Szabo, "Effect of librational motion on fluorescence depolarization and nuclear magnetic resonance relaxation in macromolecules and membranes," Biophys. J. 30, 489-506 (1980).

${ }^{60}$ G. Lipari and A. Szabo, "Model-free approach to the interpretation of nuclear magnetic resonance relaxation in macromolecules. 1. Theory and range of validity," J. Am. Chem. Soc. 104, 4546-4559 (1982).

${ }^{61}$ H.-S. Tan, I. R. Piletic, and M. D. Fayer, "Orientational dynamics of water confined on a nanometer length scale in reverse micelles," J. Chem. Phys. 122, 174501 (2005).

${ }^{62}$ D. E. Moilanen, E. E. Fenn, Y. S. Lin, J. L. Skinner, B. Bagchi, and M. D. Fayer, "Water inertial reorientation: Hydrogen bond strength and the angular potential," Proc. Natl. Acad. Sci. U. S. A. 105, 5295-5300 (2008).

${ }^{63}$ D. Laage and J. T. Hynes, "A molecular jump mechanism of water reorientation," Science 311, 832-835 (2006)

${ }^{64}$ D. Laage and J. T. Hynes, "On the molecular mechanism of water reorientation,” J. Phys. Chem. B 112, 14230-14242 (2008).

${ }^{65}$ K. Kwak, S. Park, I. J. Finkelstein, and M. D. Fayer, "Frequency-frequency correlation functions and apodization in two-dimensional infrared vibrational echo spectroscopy: A new approach," J. Chem. Phys. 127, 124503 (2007)

${ }^{66} \mathrm{~K}$. Kwak, D. E. Rosenfeld, and M. D. Fayer, "Taking apart the twodimensional infrared vibrational echo spectra: More information and elimination of distortions," J. Chem. Phys. 128, 204505 (2008).

${ }^{67}$ R. Kubo, in Fluctuation, Relaxation and Resonance in Magnetic Systems, edited by D. Ter Haar (Oliver and Boyd, London, 1961).

${ }^{68}$ P. L. Kramer, J. Nishida, C. H. Giammanco, A. Tamimi, and M. D. Fayer, "Observation and theory of reorientation-induced spectral diffusion in polarization-selective 2D IR spectroscopy," J. Chem. Phys. 142, 184505 (2015).

${ }^{69}$ P. L. Kramer, J. Nishida, and M. D. Fayer, "Separation of experimental 2D IR frequency-frequency correlation functions into structural and reorientationinduced contributions," J. Chem. Phys. 143, 124505 (2015).

${ }^{70}$ C. H. Giammanco, P. L. Kramer, S. A. Yamada, J. Nishida, A. Tamimi, and M. D. Fayer, "Carbon dioxide in an ionic liquid: Structural and rotational dynamics," J. Chem. Phys. 144, 104506 (2016)

${ }^{71}$ A. A. Vartia, K. R. Mitchell-Koch, G. Stirnemann, D. Laage, and W. H. Thompson, "On the reorientation and hydrogen-bond dynamics of alcohols," J. Phys. Chem. B 115, 12173-12178 (2011).

${ }^{72}$ S. Cerveny, F. Mallamace, J. Swenson, M. Vogel, and L. Xu, "Confined water as model of supercooled water," Chem. Rev. 116, 7608-7625 (2016).

${ }^{73}$ D. Laage, T. Elsaesser, and J. T. Hynes, "Water dynamics in the hydration shells of biomolecules," Chem. Rev. (2017).

${ }^{74}$ V. Malgras, S. Tominaka, J. W. Ryan, J. Henzie, T. Takei, K. Ohara, and Y. Yamauchi, "Observation of quantum confinement in monodisperse methylammonium lead halide perovskite nanocrystals embedded in mesoporous silica,” J. Am. Chem. Soc. 138, 13874-13881 (2016). 